\title{
Downlink Transmission of Broadband OFCDM Systems-Part V: Code Assignment
}

\author{
Yiqing Zhou, Member, IEEE, Jiangzhou Wang, Senior Member, IEEE, and Tung-Sang Ng, Fellow, IEEE
}

\begin{abstract}
To provide high speed mobile services, orthogonal multi-code transmission is employed in the orthogonal frequency and code division multiplexing (OFCDM) system. However, in a wireless frequency selective channel either with or without Doppler shift, code orthogonality is distorted and multi-code interference (MCI) is caused. The variance of the MCI is dependent on which codes to be used. This paper investigates the code assignment in the OFCDM system, aiming to mitigate the MCI. The code assignment can be explored in terms of the code distance defined in this paper. Generally, the codes with large code distances to each other should be assigned with high priority. Moreover, to avoid large interference from the powerful code-multiplexed pilot channel, the time spreading code adjacent to the pilot code in the code tree [9] is suggested not be used when the time domain spreading factor is large. The performance of the proposed non-sequential code assignment scheme is analytically evaluated. It has been shown that the system performance can be improved significantly by the non-sequential scheme under various channel conditions, especially when a low to medium system load is assumed.
\end{abstract}

Index Terms-Code assignment, OFDM, two-dimensional spreading, multi-code interference, hybrid detection, Doppler shift.

\section{INTRODUCTION}

$\mathbf{T}$ HE main purpose of the $4^{\text {th }}$ generation $(4 \mathrm{G})$ mobile communication is to provide high rate data services up to $100 \mathrm{Mbps}$, especially in the downlink over wide coverage. Various wireless access schemes [1-3] have been proposed for the broadband downlink transmission in $4 \mathrm{G}$ systems. Single carrier CDMA is not suitable over a broadband channel, due to too much multipath interference (MPI) [1]. On the other hand, although orthogonal frequency division multiplexing (OFDM) is robust to MPI [2], it dose not have coherent frequency diversity. Moreover, in mobile cellular systems, OFDM suffers from adjacent cell interference unless the same sub-carriers are not used among adjacent cells. Thus, spreading has been introduced to OFDM to provide frequency diversity and facilitate one-cell frequency reuse in a cellular environment. Combining OFDM with two-dimensional (2-D) spreading (time and frequency domain spreading) [4-6], an orthogonal frequency and code division multiplexing (OFCDM) system has been proposed for the downlink transmission in future $4 \mathrm{G}$ networks. Based on OFDM, OFCDM provides not only advantages of OFDM, but also additional benefits by means

Manuscript received April 12, 2007; revised August 4, 2007; accepted October 8,2007 . The associate editor coordinating the review of this paper and approving it for publication was X. Wang.

Y. Q. Zhou and T. S. Ng are with the Department of Electrical and Electronic Engineering, University of Hong Kong, Pokfulam Road, Hong Kong (e-mail: \{yqzhou, tsng\}@eee.hku.hk).

J. Wang is with the Department of Electronics, University of Kent, Canterbury, Kent CT2 7NT, United Kingdom (e-mail: j.z.wang@kent.ac.uk). Digital Object Identifier 10.1109/T-WC.2008.070392 of 2-D spreading. For example, frequency diversity gain can be achieved through frequency domain spreading due to the different fading experienced by sub-carriers in a broadband channel. Furthermore, with the introduction of time domain spreading, the system can provide flexible transmission rates. The time and frequency domain spreading factors, $N_{T}$ and $N_{F}$, can be changed flexibly to provide variable spreading factor (VSF) in order for the system to work in different cell environments and channel conditions.

Assuming a 2-D spreading factor of $N=N_{T} \times N_{F}$, each code channel in OFCDM can only convey $1 / N$ data symbols as many as that without spreading. In order to achieve higher data rates, the OFCDM system employs multi-code transmission, which was proposed a while ago for DS-CDMA communication [7-8] as a method to support high variable transmission rates. In the OFCDM downlink, the mobile users are served by packet switch (or time multiplexing). At any time instant, only one user can access the network. Thus, multiple code channels can be assigned to a single user to increase the transmission rate. In this case, there is no multiple user (or multiple access) interference. However, interference among the multiple code channels used by one user may occur. Using orthogonal variable spreading factor (OVSF) codes [9] as the spreading codes, the multiple code channels can be kept orthogonal to each other at the receiver if the channel is constant in both time and frequency domains, such as an additive white gaussian noise (AWGN) or static flat fading channel. However, in a broadband wireless channel, the code orthogonality at the receiver no longer maintains in the time domain because of the Doppler shift or in the frequency domain because of different fading among subcarriers. For example, at a carrier frequency of $5 \mathrm{GHz}$, when a mobile user moves at a speed of $300 \mathrm{~km} / \mathrm{hour}$, the resultant maximum Doppler shift can be as high as $1500 \mathrm{~Hz}$. Even though a short packet length of $0.5 \mathrm{~ms}$ is used in the OFCDM system, the channel variation in one packet duration is not negligible. Hence, multi-code interference (MCI) will occur in the time domain. Furthermore, in the frequency domain, the orthogonality between code channels will be distorted by frequency selectivity due to multipath propagation, so severe MCI also occurs. Therefore, in a broadband channel, all twodimensionally spreading multi-code channels in the OFCDM system interfere with each other and the resultant 2-D MCI is present. Finally, the variance of the MCI generated between any pair of code channels is dependent on which codes to be used. Use of some codes for channel spreading may result in larger MCI than that of others. In order to improve the system performance, MCI should be cancelled out as much as possible. The objective of this paper is to study code 


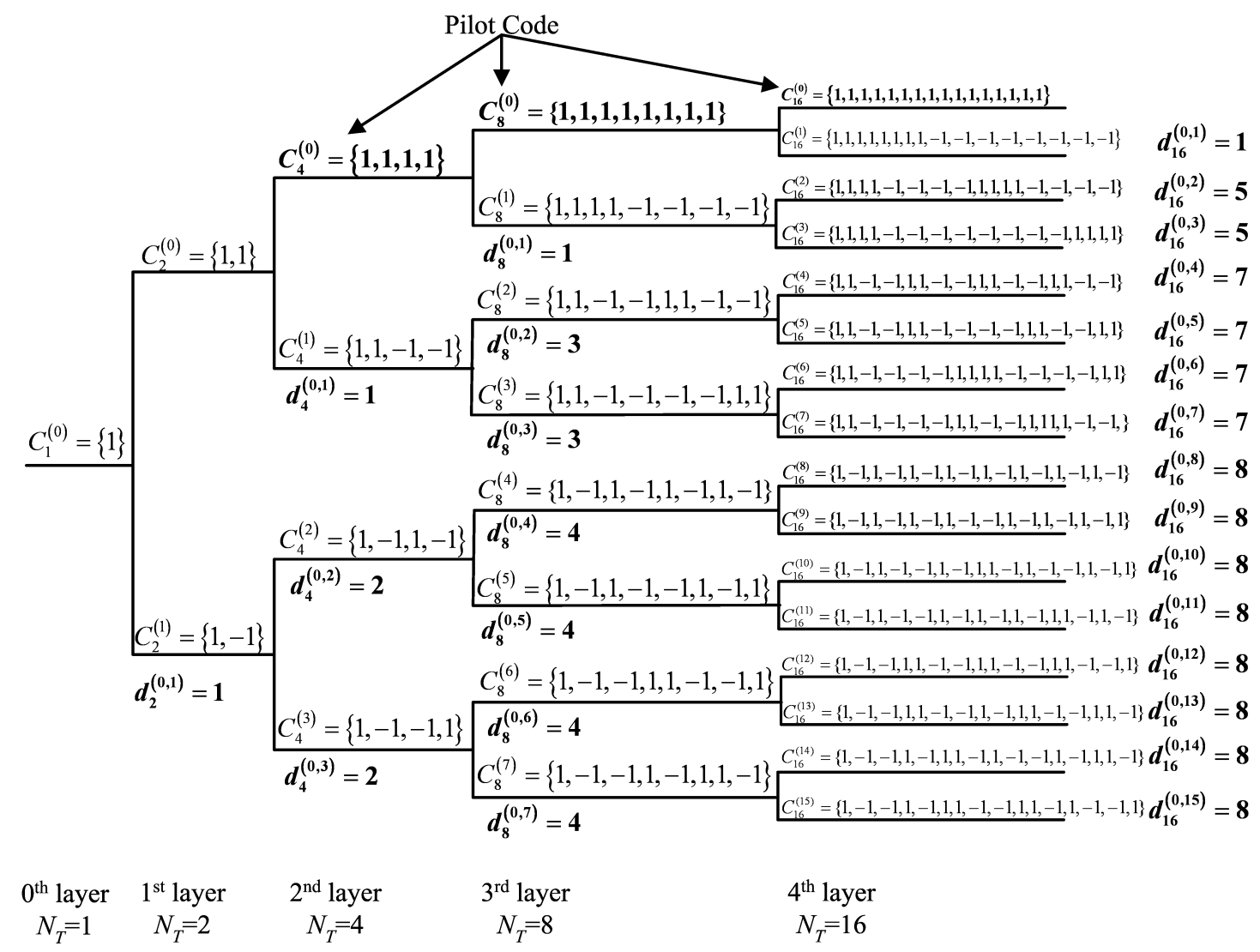

Fig. 1. OVSF code tree.

assignment to allocate appropriate codes for data transmissions so that the MCI on all the code channels can be mitigated.

Although code assignment has been studied in conventional DS-CDMA systems [10-13], these studies are focused on managing the code resource. In these studies, the physical layer performance was not studied and the channel was assumed to be flat fading so that there is no MCI among code channels. However, in the broadband OFCDM system, MCI is present and degrades the system performance. Therefore, it is necessary to investigate code assignment and study its physical layer performance in order to minimize its effect to system performance. Nevertheless, so far little research has been done on the code assignment for the multi-code OFCDM system. Code assignment was mentioned in [6] and [14], where time domain spreading was proposed to be used with higher priority than frequency domain spreading. However, there was no discussion on how to assign different codes within time or frequency domain. Generally, codes are simply assigned sequentially according to their code index on the code tree [9]. Recently, a code assignment scheme was presented in [15] for frequency domain spreading, which allocated OVSF codes having a different ancestor code to users. In this paper, the relationship between the code assignment scheme and the MCI will be studied and a non-sequential code assignment scheme for both time and frequency domain spreading will be proposed for the OFCDM system to reduce the MCI at receiver.

The rest of this paper is organized as follows. Section II describes the 2-D spreading codes, defines the code distance and introduces the system structure. Then the decision variable is derived in Section III and the non-sequential code assignment schemes are proposed in Section IV. Numerical results are shown in Section $\mathrm{V}$, where the system performance is semi-analytically evaluated. The performance of the proposed non-sequential code assignment scheme is investigated extensively with various channel conditions. Finally, conclusions are drawn in the last section.

\section{SYSTEM DESCRIPTION}

\section{A. 2-D Spreading Codes}

In the OFCDM system, data channels are 2-D spread with a spreading factor of $N=N_{T} \times N_{F}$, while the code-multiplexed pilot channel is one dimensionally spread only in the time domain with a spreading factor of $N_{T}$. Without loss of generality, the all " +1 " OVSF code, i.e., $C_{N_{T}}^{(0)}$, is assigned to the pilot channel, as shown in Fig. 1. Since the numbers of orthogonal codes are $N_{T}$ and $N_{F}$ in time domain and frequency domain, respectively, there are $\left(N_{T}-1\right) N_{F}$ codes available for 2-D data channels. Assuming that $K$ out of the $\left(N_{T}-1\right) N_{F}$ code channels are used for data transmission, the system load is defined by $K /\left[\left(N_{T}-1\right) N_{F}\right]$. A 2-D spreading code is denoted as $\left\{C_{N_{T}}^{\left(k_{T}\right)}, C_{N_{F}}^{\left(k_{F}\right)}\right\}$, where $C_{N_{T}}^{\left(k_{T}\right)}=\left\{c_{N_{T}, 0}^{\left(k_{T}\right)}, \cdots, c_{N_{T}, N_{T}-1}^{\left(k_{T}\right)}\right\}$ is the time domain spreading code with length of $N_{T}$ and $C_{N_{F}}^{\left(k_{F}\right)}=\left\{c_{N_{F}, 0}^{\left(k_{F}\right)}, \cdots, c_{N_{F}, N_{F}-1}^{\left(k_{F}\right)}\right\}$ is the frequency domain spreading code with length of $N_{F}$. Both $C_{N_{T}}^{\left(k_{T}\right)}$ and $C_{N_{F}}^{\left(k_{F}\right)}$ are OVSF codes and can be generated by a code tree. The generation of the time domain spreading codes is shown in Fig. 1. Similarly, the frequency domain spreading codes can be generated by using the tree as well but with the 


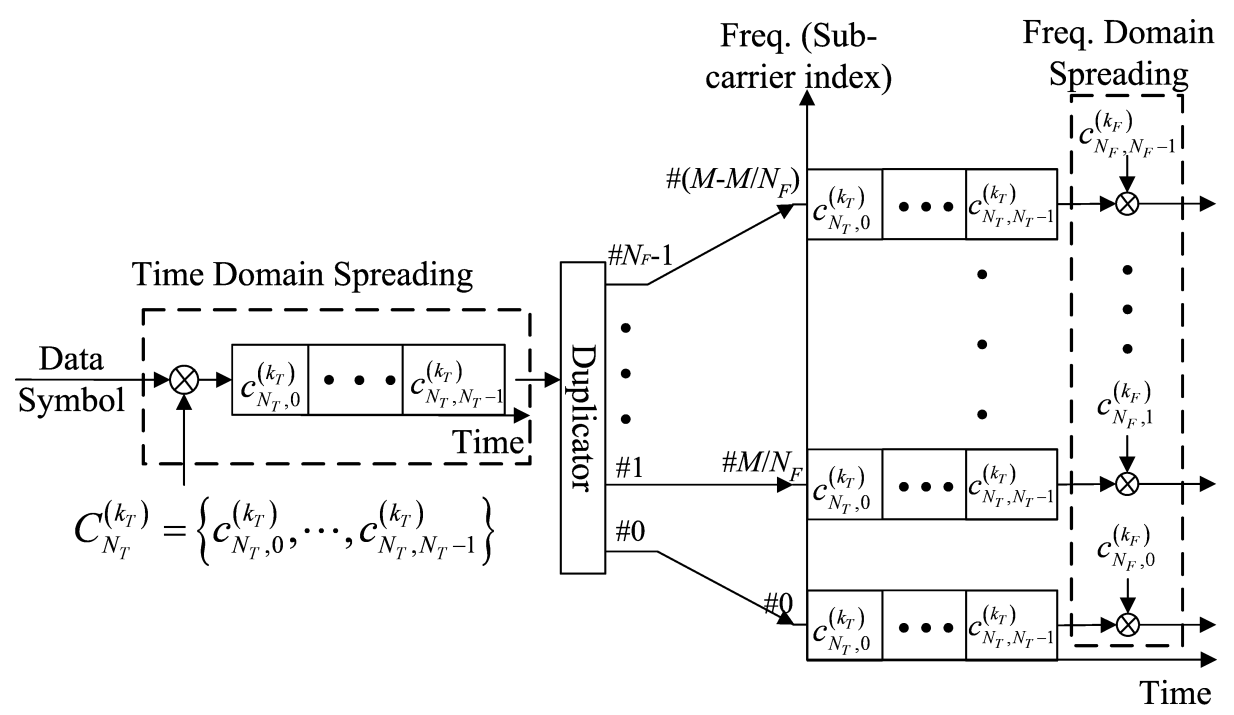

Fig. 2. Two-dimensional spreading.

substitution of $N_{T}$ by $N_{F}$ and the use of the pilot code as a data code. In the OVSF code tree, $C_{N_{T}}^{\left(k_{T}\right)}$ stands for the $k_{T}^{\text {th }}\left(k_{T}=1, \ldots, N_{T}-1\right)$ code on the $\left(\log _{2} N_{T}\right)^{\text {th }}$ layer (see Fig. 1), while $C_{N_{F}}^{\left(k_{F}\right)}$ stands for the $k_{F}^{\text {th }}\left(k_{F}=0, \ldots, N_{F}-1\right)$ code on the $\left(\log _{2} N_{F}\right)^{\text {th }}$ layer.

The distance of two OVSF codes, $C_{N_{T}}^{\left(k_{T}\right)}$ and $C_{N_{T}}^{\left(\bar{k}_{T}\right)}$, is denoted as $d_{N_{T}}^{\left(k_{T}, \bar{k}_{T}\right)}$, defined as $N_{T} / 2+1$ subtracted by the minimum length of strings of consecutive 1's or -1 's in the code product, $C_{N_{T}}^{\left(k_{T}\right)} \cdot C_{N_{T}}^{\left(\bar{k}_{T}\right)}$, which is given by

$$
\begin{aligned}
& C_{N_{T}}^{\left(k_{T}\right)} \cdot C_{N_{T}}^{\left(\bar{k}_{T}\right)}=\left\{c_{N_{T}, 0}^{\left(k_{T}\right)} \cdot c_{N_{T}, 0}^{\left(\bar{k}_{T}\right)}, c_{N_{T}, 1}^{\left(k_{T}\right)} \cdot c_{N_{T}, 1}^{\left(\bar{k}_{T}\right)},\right. \\
& \left.\ldots, c_{N_{T}, N_{T}-1}^{\left(k_{T}\right)} \cdot c_{N_{T}, N_{T}-1}^{\left(\bar{k}_{T}\right)}\right\}
\end{aligned}
$$

For example, consider the distance between the pilot code $C_{16}^{(0)}$ and $C_{16}^{(1)}$. Since $C_{16}^{(0)}$ is the all " +1 " code, the code product $C_{16}^{(0)} \cdot C_{16}^{(1)}$ equals $C_{16}^{(1)}$. There are two equal strings of eight consecutive 1's and eight consecutive -1's in $C_{16}^{(1)}$. Therefore, the distance is one $\left(d_{16}^{(0,1)}=9-8=1\right)$. Furthermore, consider the distance between $C_{16}^{(0)}$ and $C_{16}^{(9)}$. There is one string of two consecutive -1's and 14 strings of single 1's or -1 's in $C_{16}^{(9)}$. Hence, the distance between $C_{16}^{(0)}$ and $C_{16}^{(9)}$ is $d_{16}^{(0,9)}=9-\min \{1,2\}=8$. The detailed distances between the pilot code and other codes for different spreading factors are shown in Fig. 1. It can be seen that the code with a minimum distance to the pilot code is always $C_{N_{T}}^{(1)}$. Note that the definition of the code distance here is different from that of the Hamming distance in the coding theory.

Suppose that the 2-D code $\left\{C_{N_{T}}^{\left(\bar{k}_{T}\right)}, C_{N_{F}}^{\left(\bar{k}_{F}\right)}\right\}$ is the code of interest. Excluding $\left\{C_{N_{T}}^{\left(\bar{k}_{T}\right)}, C_{N_{F}}^{\left(\bar{k}_{F}\right)}\right\}$, the rest of the codes can be divided into two subsets: one set, $\Omega_{T}=\left\{\left\{C_{N_{T}}^{\left(k_{T}\right)}, C_{N_{F}}^{\left.k_{F}\right)}\right\}\right.$ | $\left.k_{T} \neq \bar{k}_{T}\right\}$, with different time domain spreading codes from $C_{N_{T}}^{\left(\bar{k}_{T}\right)}$, and another set, $\Omega_{F}=\left\{\left\{C_{N_{T}}^{\left(\bar{k}_{T}\right)}, C_{N_{F}}^{\left.k_{F}\right)}\right\} \mid k_{F} \neq \bar{k}_{F}\right\}$, with the same time domain spreading code but different frequency domain spreading codes from $C_{N_{F}}^{\left(\bar{k}_{F}\right)}$. The codes in $\Omega_{T}$ must be orthogonal to $\left\{C_{N_{T}}^{\left(\bar{k}_{T}\right)}, C_{N_{F}}^{\left(\bar{k}_{F}\right)}\right\}$ in the time domain, while the codes in $\Omega_{F}$ are orthogonal to $\left\{C_{N_{T}}^{\left(\bar{k}_{T}\right)}, C_{N_{F}}^{\left(\bar{k}_{F}\right)}\right\}$ only in the frequency domain.

\section{B. System Structure and Channel Model}

Consider the $k^{\text {th }}$ data code channel using QPSK and 2-D spreading with the code $\left\{C_{N_{T}}^{\left(k_{T}\right)}, C_{N_{F}}^{\left(k_{F}\right)}\right\}$. As shown in Fig. 2, in the 2-D spreading, each data symbol on the $k^{\text {th }}$ data code channel is firstly spread by the time domain spreading code $C_{N_{T}}^{\left(k_{T}\right)}$. Then, the time domain spread signal is duplicated into $N_{F}$ interleaved copies in the frequency domain and multiplied by the frequency domain spreading code $C_{N_{F}}^{\left(k_{F}\right)}$. Therefore, totally $N=N_{T} \times N_{F}$ spread chips per data symbol are obtained by means of the 2-D spreading. At the same time, on each sub-carrier, known QPSK modulated pilot symbols are spread with $N_{T}$ chips only in time domain. The spread signals on all $K$ data code channels and the pilot channel will be added together by a code multiplexer, then transmitted on the $N_{F}$ interleaved sub-carriers in parallel. Practically, each data packet is composed of dozens (e.g., 48 in [6]) of OFCDM symbols (or time chips), and a large number (e.g., 1024) of sub-carriers are employed. Assuming that there are totally $M$ sub-carriers in the OFCDM system, $M / N_{F}$ data symbols can be transmitted simultaneously in the frequency domain using all sub-carriers. With a block interleaver, the first data symbol uses the $0^{\text {th }},\left(M / N_{F}\right)^{\text {th }}, \ldots,\left(M-\left(M / N_{F}\right)\right)^{\text {th }}$ subcarriers, the second one uses the $1^{\text {st }},\left(M / N_{F}+1\right)^{\text {th }}, \ldots$, $\left(M-\left(M / N_{F}\right)+1\right)^{\mathrm{th}}$ sub-carriers, and so on. Therefore, $N_{T}$ OFCDM time chips and $N_{F}$ interleaved sub-carriers carrying the same data symbol are concerned for each data symbol. The transmitted signal is given by

$$
S(t)=\sum_{i=0}^{N_{T}-1} \sum_{m=0}^{N_{F}-1}\left[\begin{array}{l}
\left(\sqrt{P} \sum_{k=0}^{K-1} d_{k} c_{N_{T}, i}^{\left(k_{T}\right)} c_{N_{F}, m}^{\left(k_{F}\right)}+\sqrt{\beta P} d_{P}\right) \\
\cdot e^{j 2 \pi f_{m}(t-i T)} p(t-i T)
\end{array}\right]
$$

where $P$ is the signal power of one data channel, $K$ $\left(K \leq\left(N_{T}-1\right) N_{F}\right)$ is the number of data code channels, $d_{k}$ is the data symbol of the $k^{\text {th }}$ data channel with $\left|d_{k}\right|^{2}=1$, $c_{N_{T}, i}^{\left(k_{T}\right)}$ and $c_{N_{F}, m}^{\left(k_{F}\right)}$ are chips of the time domain and the frequency domain spreading codes, respectively, $\beta$ is the power 


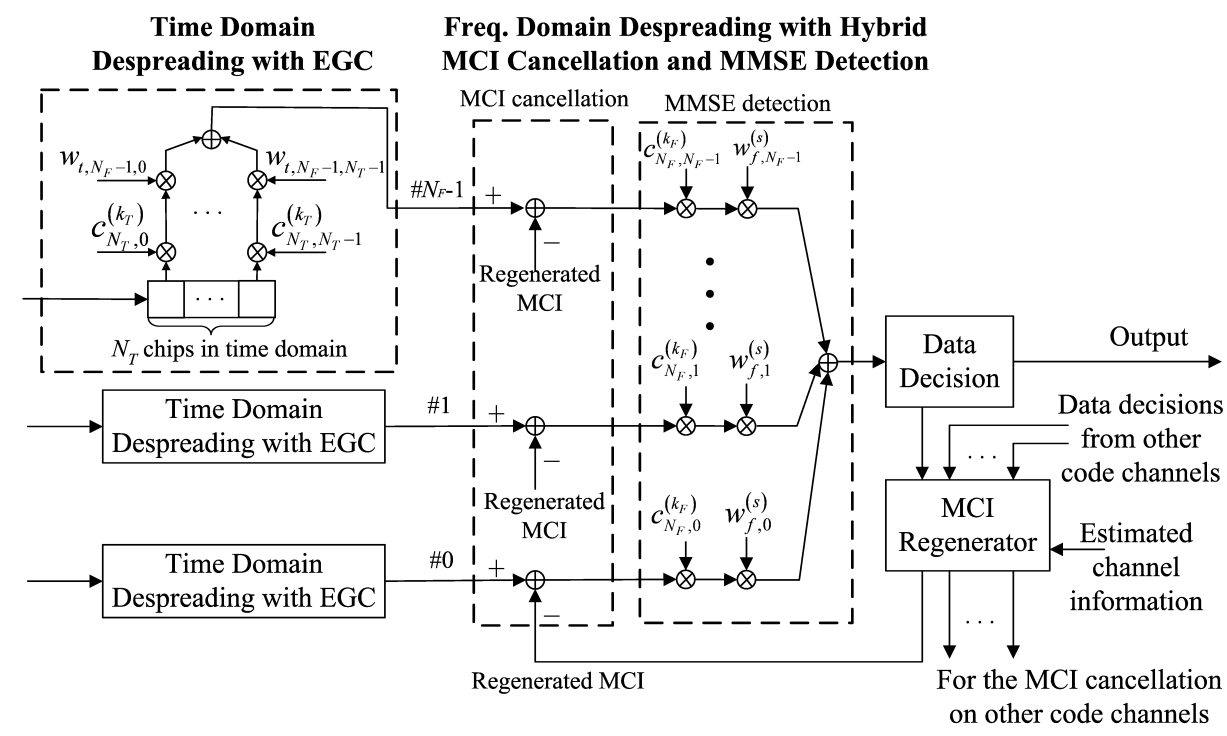

Fig. 3. Two-dimensional despreading and combining.

ratio of pilot to one data channel, $d_{P}$ is the known pilot symbol with $\left|d_{P}\right|^{2}=1, f_{m}=m M /\left(N_{F} T_{e}\right)$ represents the baseband equivalent frequency of the $\left(m M / N_{F}\right)^{\text {th }}$ sub-carrier, or the $m^{\text {th }}$ interleaved sub-carrier for the concerned data symbol, where $T_{e}$ stands for the effective duration of an OFCDM symbol, $T=T_{e}+T_{g}$ is the complete duration including the guard interval $T_{g}$, and $p(t)$ is the impulse response (unitary rectangular) of the pulse shaping filter.

The transmitted signal passes through a broadband wireless channel, which is highly frequency selective. However, in the mutli-carrier OFCDM system, the signal transmitted on each sub-carrier experiences a flat fading channel. Assuming perfect suppression of multipath by the guard interval $T_{g}$, a parallel flat fading multichannel model can be employed [16] instead of the conventional multipath model. Let $H_{m}(t)$ represent the complex channel fading on the $\left(m M / N_{F}\right)^{\text {th }}$ sub-carrier, or the $m^{\text {th }}$ interleaved sub-carrier. The amplitude and phase of $H_{m}(t)$ are Rayleigh distributed with $E\left\{\left|H_{m}(t)\right|^{2}\right\}=1$ and uniformly distributed in $[0,2 \pi)$, respectively. The parallel multichannel model is characterized by the time-frequency joint correlation function of $H_{m}(t)$. Assuming a U-shape Doppler spectrum and an exponentially distributed delay time, the joint correlation function of the corresponding parallel flat fading multichannel is given by [17]

$$
\begin{aligned}
\rho\left(m_{0}, m_{1}, t_{0}, t_{1}\right) & =\frac{E\left\{H_{m_{0}}\left(t_{0}\right) H_{m_{1}}^{*}\left(t_{1}\right)\right\}}{E\left\{\left|H_{m}(t)\right|^{2}\right\}} \\
& =\frac{J_{0}\left(2 \pi f_{D}\left|t_{0}-t_{1}\right|\right)}{\sqrt{1+\left[\left|m_{0}-m_{1}\right| M /\left(N_{F} T_{e} \Delta f_{c}\right)\right]^{2}}}
\end{aligned}
$$

where " $*$ " stands for the complex conjugate, $\left|t_{0}-t_{1}\right|$ is the time difference, $\left|m_{0}-m_{1}\right| M /\left(N_{F} T_{e}\right)$ is the frequency separation between the $m_{0}^{\text {th }}$ and $m_{1}^{\text {th }}$ interleaved sub-carriers, $f_{D}$ is the Doppler frequency shift, $\Delta f_{c}$ is the channel coherence bandwidth, and $J_{0}(\cdot)$ is the zeroth-order Bessel function of the first kind. It can be seen from (3) that the characteristics of the channel is determined by the Doppler shift $f_{D}$ and and the coherence bandwidth $\Delta f_{c}$, where $\Delta f_{c} \approx 1 / \tau_{r m s}$ and $\tau_{r m s}$ is the root-mean-square delay of the corresponding multipath channel. Moreover, by setting the frequency difference or time difference to zero, the time correlation and frequency correlation can be obtained as

$$
\rho_{t}\left(t_{0}, t_{1}\right)=\frac{E\left\{H_{m}\left(t_{0}\right) H_{m}^{*}\left(t_{1}\right)\right\}}{E\left\{\left|H_{m}(t)\right|^{2}\right\}}=J_{0}\left(2 \pi f_{D}\left|t_{0}-t_{1}\right|\right)
$$

and

$$
\begin{aligned}
\rho_{f}\left(m_{0}, m_{1}\right) & =\frac{E\left\{H_{m_{0}}(t) H_{m_{1}}^{*}(t)\right\}}{E\left\{\left|H_{m}(t)\right|^{2}\right\}} \\
& =1 / \sqrt{1+\left[\left|m_{0}-m_{1}\right| M /\left(N_{F} T_{e} \Delta f_{c}\right)\right]^{2}},
\end{aligned}
$$

respectively.

At the receiver, the signals transmitted on parallel subcarriers will be firstly down-converted to base-band signals. Channel estimation is carried out on each sub-carrier by using the code-multiplexed pilot channel [18-19]. The preliminary channel estimations are firstly recovered by despreading the received signal using the pilot code in time domain. If each data packet is composed of $N_{D}$ OFCDM time chips, with a pilot spreading factor of $N_{T}$, there are $N_{D} / N_{T}$ preliminary channel estimations in one packet. These preliminary estimations will be used to predict the channel fading factor on each OFCDM time chip by means of linear interpolation. Note that this linear interpolation channel estimation works effectively only when the Doppler shift is small. When the Doppler shift is large, it is difficult to obtain accurate channel estimation on each OFCDM time chip. Given channel information, 2-D despreading can be carried out as shown in Fig. 3. The received signal on each sub-carrier is firstly processed by the time domain despreading with equal gain combining (EGC), where $N_{T}$ time chips are multiplied by the time domain spreading code $C_{N_{T}}^{\left(k_{T}\right)}=\left\{c_{N_{T}, 0}^{\left(k_{T}\right)}, \ldots, c_{N_{T}, N_{T}-1}^{\left(k_{T}\right)}\right\}$ and the EGC weights $\left\{w_{t, N_{F}-1,0}, \ldots, w_{t, N_{F}-1, N_{T}-1}\right\}$ (or channel phases), then summed over the $N_{T}$ chips. The simple EGC is considered because the MCI in time domain (MCI-T) is relatively small. Although MCI-T increases with the Doppler shift $f_{D}, f_{D}$ is limited by the moving speed of mobile stations 


$$
\begin{aligned}
r_{\bar{m}, \bar{i}} & =\frac{1}{T_{e}} \int_{\bar{i} T}^{\bar{i} T+T_{e}} r(t) e^{-j 2 \pi f_{\bar{m}}(t-\bar{i} T)} p\left(\bar{i} T+T_{e}-t\right) d t \\
& =\frac{1}{T_{e}} \int_{\bar{i} T}^{\bar{i} T+T_{e}}\left[\sum_{m=0}^{N_{F}-1} \sum_{i=0}^{N_{T}-1}\left[\begin{array}{l}
\left.\left.\left(\sqrt{P} \sum_{k=0}^{K-1} d_{k} c_{N_{T}, i}^{\left(k_{T}\right)} c_{N_{F}, m}^{\left(k_{F}\right)}+\sqrt{\beta P} d_{P}\right)\right] H_{m}(t)+\eta(t)\right] e^{-j 2 \pi f_{m}(t-i T)} p(t-\bar{i} T)
\end{array}\right]\right. \\
& \approx \sqrt{P} \sum_{k=0}^{K-1} d_{k} c_{N_{T}, \bar{i}}^{\left(k_{T}\right)} c_{N_{F}, \bar{m}}^{\left(k_{F}\right)} \lambda_{\bar{m}, \bar{i}}+\sqrt{\beta P} d_{P} \lambda_{\bar{m}, \bar{i}}+\eta_{\bar{m}, \bar{i}}
\end{aligned}
$$

which is generally less than $2000 \mathrm{~Hz}$ in practice with a carrier frequency of $5 \mathrm{GHz}$. Therefore, the MCI-T in a short packet duration $(0.5 \mathrm{~ms})$ is less serious than the $\mathrm{MCI}$ in frequency domain (MCI-F), which is caused by different channel fading on interleaved sub-carriers in a highly frequency selective channel. To suppress the serious MCI-F, frequency domain despreading is carried out with superior hybrid MCI cancellation and minimum mean square error (MMSE) detection [18]. The basic idea of the hybrid detection is illustrated in Fig. 3 , where $C_{N_{F}}^{\left(k_{F}\right)}=\left\{c_{N_{F}, 0}^{\left(k_{F}\right)}, \ldots, c_{N_{F}, N_{F}-1}^{\left(k_{F}\right)}\right\}$ is the frequency domain spreading code and $\left\{w_{f, 0}^{(s)}, \ldots, w_{f, N_{F}-1}^{(s)}\right\}$ (detailed in the next section) is the MMSE weights at the $s^{\text {th }}$ stage of the hybrid detection. The $0^{\text {th }}$ stage of the hybrid detection is the conventional MMSE (or pure MMSE) combining without MCI cancellation. At this stage, the regenerated MCI-F is zero since there are no previous data decisions. The signals at the output of the time domain despreader with EGC are processed directly by the frequency domain despreader with MMSE. Then data decisions are obtained. The tentative data decisions at the $0^{\text {th }}$ stage input to a MCI regenerator with channel information (estimated by using the pilot channel), so that the MCI-F can be regenerated. At the $1^{\text {st }}$ stage of the hybrid detection, the regenerated MCI-F from the $0^{\text {th }}$ stage is fed back and cancelled out from the output of the time domain despreader. The signal after MCI cancellation is further despread in the frequency domain with MMSE combining. Since the MMSE weight is related to the input signal power, it should be updated stage by stage due to the reduction of MCI at each stage. After the hybrid detection, new data decisions are obtained for the $1^{\text {st }}$ stage. Similarly, they should be used to regenerate the MCI-F for MCI cancellation at the next stage. This iterative operation will go on until a specified number of stages is reached. Although the hybrid detection in the OFCDM system has been shown to be an effective technique to reduce MCI, it will be shown in Section V that a good code assignment scheme can help reduce MCI further.

\section{DERIVATION OF MCI}

\section{A. Decision Variables}

In order to propose an effective code assignment scheme in the OFCDM system, the MCI generated from different code channels are investigated first. After passing through the broadband channel and corrupted by a complex AWGN, $\eta(t)$, the received baseband signal is denoted as $r(t)$. By setting $T_{g}$ larger than the maximum channel delay, there is no interference from adjacent OFCDM time chips. Thus, the output of the matched filter on the $\left(\bar{m} M / N_{F}\right)^{\text {th }}$ sub-carrier, or the $\bar{m}^{\text {th }}$ interleaved sub-carrier, in the $\bar{i}^{\text {th }}$ OFCDM time chip duration can be expressed as (6), where

$$
\lambda_{\bar{m}, \bar{i}}=\frac{1}{T_{e}} \int_{\bar{i} T}^{\bar{i} T+T_{e}} H_{\bar{m}}(t+\bar{i} T) d t
$$

is the channel fading factor on the $\bar{m}^{\text {th }}$ interleaved sub-carrier, $\eta_{\bar{m}, \bar{i}}$ is the noise component with zero mean and variance $\sigma_{n}^{2}=N_{0} / T_{e}$, and $N_{0}$ is the power spectral density of $\eta(t)$. Note that with a broad bandwidth around $100 \mathrm{MHz}$ and a total number of sub-carriers of 1024, the frequency spacing between two adjacent sub-carriers is nearly $100 \mathrm{KHz}$. In this case, the effect of the inter-carrier interference (ICI) caused by Doppler shift is insignificant as long as $f_{D}$ is less than $5 \mathrm{KHz}$ [19-20]. Therefore, ICI term is ignored in $r_{\bar{m}, \bar{i}}$.

The output of the time domain despreader with the EGC combining is then given by (8) (shown on the top of next page), where $w_{t, \bar{m}, \bar{i}}=\hat{\lambda}_{\bar{m}, \bar{i}}^{*} /\left|\hat{\lambda}_{\bar{m}, \bar{i}}\right|$ is the EGC weight, $\hat{\lambda}_{\bar{m}, \bar{i}}$ is the estimated channel information using the code-multiplexed pilot channel, $S_{\bar{k}, \bar{m}}$ is the useful signal from the reference code, $M C I_{\bar{k}, \bar{m}}^{(F)}$ is the MCI-F, $M C I_{\bar{k}, \bar{m}}^{(T)}$ is the MCI-T caused by the pilot code and other data codes in $\Omega_{T}$, and $\eta_{\bar{k}, \bar{m}}$ is the background noise. Since the hybrid MCI cancellation and MMSE detection is employed in the frequency domain, at the $s^{\text {th }}$ stage, the regenerated MCI-F for the $\bar{k}_{\text {th }}$ code channel on the $\bar{m}_{\text {th }}$ sub-carrier is given by

$$
Q_{\bar{k}, \bar{m}}^{(F)}(s)=\sqrt{P} \sum_{k \in \Omega_{F}} d_{k}^{(s-1)} c_{N_{F}, \bar{m}}^{\left(k_{F}\right)}\left(\sum_{\bar{i}=0}^{N_{T}-1} \lambda_{\bar{m}, \bar{i}} w_{t, \bar{m}, \bar{i}}\right)
$$

where $d_{k}^{(s-1)}$ is the data decision at the previous stage. Note that the data decisions are not available at the zeroth stage (i.e., $d_{k}^{(s-1)}=0$ when $s=0$ ). Then, the output of the time domain despreader is subtracted by $Q_{\frac{(F)}{k}, m}(s)$ to carry out MCI cancellation and the resultant signal is given by

$$
\gamma_{\bar{k}, \bar{m}}^{(s)}=\gamma_{\bar{k}, \bar{m}}-Q_{\bar{k}, \bar{m}}^{(F)}(s) .
$$

$\gamma_{\bar{k}, \bar{m}}^{(s)}$ is further processed by the frequency domain despreader with the MMSE combining, and the decision variable is given by

$$
\begin{aligned}
y_{\bar{k}}^{(s)} & =\sum_{\bar{m}=0}^{N_{F}-1} \gamma_{\bar{k}, \bar{m}}^{(s)}\left[c_{N_{F}, \bar{m}}^{\left(\bar{k}_{F}\right)} w_{f, \bar{m}}^{(s)}\right] \\
& =S_{\bar{k}}^{(s)}+M C I_{\bar{k}}^{(T)}(s)+M C I_{\bar{k}}^{(F)}(s)+\eta_{\bar{k}}^{(s)}
\end{aligned}
$$




$$
\begin{aligned}
\gamma_{\bar{k}, \bar{m}}= & \sum_{\bar{i}=0}^{N_{T}-1} r \bar{m}, \bar{i} c_{N_{T}, \bar{i}}^{\left(\bar{k}_{T}\right)} w_{t, \bar{m}, \bar{i}}=\underbrace{\sqrt{P} d_{\bar{k}} c_{N_{F}, \bar{m}}^{\left(\bar{k}_{F}\right)}\left(\sum_{\bar{i}=0}^{N_{T}-1} \lambda_{\bar{m}, \bar{i}} w_{t, \bar{m}, \bar{i}}\right)}_{S_{\bar{k}, \bar{m}}}+\underbrace{\sqrt{P} \sum_{k \in \Omega_{F}} d_{k} c_{N_{F}, \bar{m}}^{\left(k_{F}\right)}\left(\sum_{\bar{i}=0}^{N_{T}-1} \lambda_{\bar{m}, \bar{i}} w_{t, \bar{m}, \bar{i}}\right)}_{M C I_{\bar{k}, \bar{m}}^{(F)}} \\
& +\underbrace{\sqrt{P} \sum_{k \in \Omega_{T}} d_{k} c_{N_{F}, \bar{m}}^{\left(k_{F}\right)}\left(\sum_{\bar{i}=0}^{N_{T}-1} \lambda_{\bar{m}, \bar{i}} w_{t, \bar{m}, \bar{i}} c_{N_{T}, \bar{i}}^{k_{T}} c_{N_{T}, \bar{i}}^{\bar{k}_{T}}\right)+\sqrt{\beta P} d_{P}\left(\sum_{\bar{i}=0}^{N_{T}-1} \lambda_{\bar{m}, \bar{i}} w_{t, \bar{m}, \bar{i}} c_{N_{T}, \bar{i}}^{\bar{k}_{T}}\right)+\eta_{\bar{k}, \bar{m}}}_{M C I_{\bar{k}, \bar{m}}^{(T)}}
\end{aligned}
$$

where $w_{f, m}^{(s)}$ is the MMSE weight, given by

$$
\begin{aligned}
w_{f, \bar{m}}^{(s)} & =\frac{E\left\{\sqrt{P} d_{\bar{k}}\left[\gamma_{\bar{k}, \bar{m}}^{(s)}\right]^{*} \mid \widehat{\lambda}_{\bar{m}, \bar{i}}\right\} c_{N_{F}, \bar{m}}^{\left(\bar{k}_{F}\right)}}{E\left\{\left|\gamma_{\bar{k}, \bar{m}}^{(s)}\right|^{2} \mid \widehat{\lambda}_{\bar{m}, \bar{i}}\right\}} \\
& =\frac{P \widehat{\lambda}_{\bar{m}, \bar{i}}^{*}}{E\left\{\left|\gamma_{\bar{k}, \bar{m}}^{(s)}\right|^{2} \mid \hat{\lambda}_{\bar{m}, \bar{i}}\right\}}
\end{aligned}
$$

In practice, the ensemble average $E\left\{\left|\gamma_{\bar{k}, \bar{m}}^{(s)}\right|^{2} \mid \widehat{\lambda}_{\bar{m}, \bar{i}}\right\}$ can be obtained by estimating with time, frequency and code averages. A detailed description of the estimation algorithm was presented in [18]. $S_{\bar{k}}^{(s)}$ is the useful signal, given by

$$
S_{\bar{k}}^{(s)}=\sqrt{P} d_{\bar{k}}\left(\sum_{\bar{m}=0}^{N_{F}-1} \sum_{\bar{i}=0}^{N_{T}-1} \lambda_{\bar{m}, \bar{i}} w_{t, \bar{m}, \bar{i}} w_{f, \bar{m}}^{(s)}\right)
$$

$M C I_{\bar{k}}^{(T)}(s)$ is the MCI-T, given by

$$
\begin{aligned}
& M C I_{\bar{k}}^{(T)}(s)=\sqrt{P} \sum_{k \in \Omega_{T}} d_{k}\left[\sum_{\bar{m}=0}^{N_{F}-1}\left(\begin{array}{l}
c_{N_{F}, \bar{m}}^{\left(k_{F}\right)} c_{N_{F}, \bar{m}}^{\left(\bar{k}_{F}\right)} w_{f, \bar{m}}^{(s)} \\
\cdot \sum_{\bar{i}=0}^{N_{T}-1} \lambda_{\bar{m}, \bar{i}} w_{t, \bar{m}, \bar{i}} c_{N_{T}, \bar{i}}^{\left(k_{T}\right)} c_{N_{T}, \bar{i}}^{\left(\bar{k}_{T}\right)}
\end{array}\right)\right] \\
& +\sqrt{\beta P} d_{P}\left[\sum_{\bar{m}=0}^{N_{F}-1} c_{N_{F}, \bar{m}}^{\left(\bar{k}_{F}\right)} w_{f, \bar{m}}^{(s)}\left(\sum_{\bar{i}=0}^{N_{T}-1} \lambda_{\bar{m}, \bar{i}} w_{t, \bar{m}, \bar{i}} c_{N_{T}, \bar{i}}^{\left(\bar{k}_{T}\right)}\right)\right]
\end{aligned}
$$

$M C I_{\bar{k}}^{(F)}(s)$ is the residual MCI-F, given by

$$
\begin{gathered}
M C I_{\bar{k}}^{(F)}(s)=\sqrt{P} \sum_{k \in \Omega_{F}} d_{k}\left[\sum_{\bar{m}=0}^{N_{F}-1}\left(\begin{array}{c}
c_{N_{F}, \bar{m}}^{\left(k_{F}\right)} c_{N_{F}, \bar{m}}^{\left(\bar{k}_{F}\right)} w_{f, \bar{m}}^{(s)} \\
\sum_{\bar{i}=0}^{N_{T}-1} \lambda_{\bar{m}, \bar{i}} w_{t, \bar{m}, \bar{i}}
\end{array}\right)\right] \\
-\sqrt{P} \sum_{k \in \Omega_{F}} d_{k}^{(s-1)}\left[\sum_{\bar{m}=0}^{N_{F}-1}\left(\begin{array}{c}
c_{N_{F}, \bar{m}}^{\left(k_{F}\right)} c_{N_{F}, \bar{m}}^{\left(\bar{k}_{F}\right)} w_{f, \bar{m}}^{(s)} \\
\cdot \sum_{\bar{i}=0}^{N_{T}-1} \widehat{\lambda}_{\bar{m}, \bar{i}} w_{t, \bar{m}, \bar{i}}
\end{array}\right)\right]
\end{gathered}
$$

and $\eta_{\bar{k}}^{(s)}$ is the background noise.

\section{B. System Configurations}

Unless noted otherwise, the system is configured as follows. The system bandwidth is $100 \mathrm{MHz}$. The number of subcarriers is $M=1024$ and the resultant sub-carrier spacing is $\Delta f=97.7 \mathrm{kHz}$. The effective OFCDM time chip duration is $10.24 \mu \mathrm{s}$, a guard interval $T_{g}$ is $2.26 \mu \mathrm{s}$, and the complete OFCDM time chip duration $T$ is $12.5 \mu \mathrm{s}$. That is, the guard interval is $18 \%$ of the complete time chip duration. Each packet is composed of $N_{D}=48$ OFCDM time chips and the packet length is $0.6 \mathrm{~ms}$. The 2-D spreading factor is set to $N=16 \times 4$. Hence, the data symbol duration is given by $T_{D}=N_{T} \cdot T=0.2 \mathrm{~ms}$. The number of data symbols in one packet is $M N_{D} K / N=768 K$, where $K$ is the number of data code channels. Moreover, a parallel flat-fading multichannel model is considered. A Doppler shift $f_{D}$ of $1000 \mathrm{~Hz}$ is assumed. Note that the channel coherence time is $\Delta T_{c} \approx 1 / f_{D}=1.0 \mathrm{~ms}$. Although $\Delta T_{c}$ is larger than the data symbol duration $\left(T_{D}=0.2 \mathrm{~ms}\right)$, these two values are comparable and the channel can be considered as fast fading. Moreover, as can be seen from (5), the correlation coefficient in frequency domain depends on the frequency separation. Given a coherence bandwidth $\Delta f_{c}$, the channel correlation between adjacent interleaved sub-carriers is used as a reference of the frequency correlation, which is set to $\rho_{f}=\rho_{f}(0,1)=1 / \sqrt{1+\left[M /\left(N_{F} T_{e} \Delta f_{c}\right)\right]^{2}} \approx 0.10$, corresponding $\Delta f_{c}=2.5 \mathrm{MHz}$ or $\tau_{r m s} \approx 0.4 \mu \mathrm{s}$. The correlated Rayleigh fading channels are generated according to [21]. The average signal-to-noise ratio (SNR) per bit is defined as $S N R_{b}=\frac{P}{2 \sigma_{n}^{2}} N(1+\beta / K)$, where the power ratio between the pilot channel and all data channels is $\beta / K=0.25$, which is near the optimum value.

\section{Code Assignment Schemes}

\section{A. Time Domain Code Assignment}

Investigate the MCI-T first. From the MCI-T in (8), it can be seen that in a static fading channel, the MCI-T equals zero since the channel fading factor $\lambda_{\bar{m}, \bar{i}}$ and the EGC weight $w_{t, \bar{m}, \bar{i}}$ are fixed in time domain. However, in a non-static fading channel, $\lambda_{\bar{m}, \bar{i}}$ and $w_{t, \bar{m}, \bar{i}}$ are not fixed in time domain so that non-zero MCI-T is caused. The more variation the channel has, the more MCI-T is caused. Therefore, the MCI-T between two channels with codes $C_{N_{T}}^{\left(\bar{k}_{T}\right)}$ and $C_{N_{T}}^{\left(k_{T}\right)}$ is mainly determined by the channel condition and the two codes. To easily show the MCI-T among different codes, the output of a simple time domain despreader without any weighting is considered, i.e., $w_{t, \bar{m}, \bar{i}} \equiv 1$ for all $\bar{m}$ and $\bar{i}$. Section $\mathrm{V}$ will show that code assignment schemes obtained with simple depreaders without weighting can work effectively in the OFCDM system with EGC weights in time domain and 


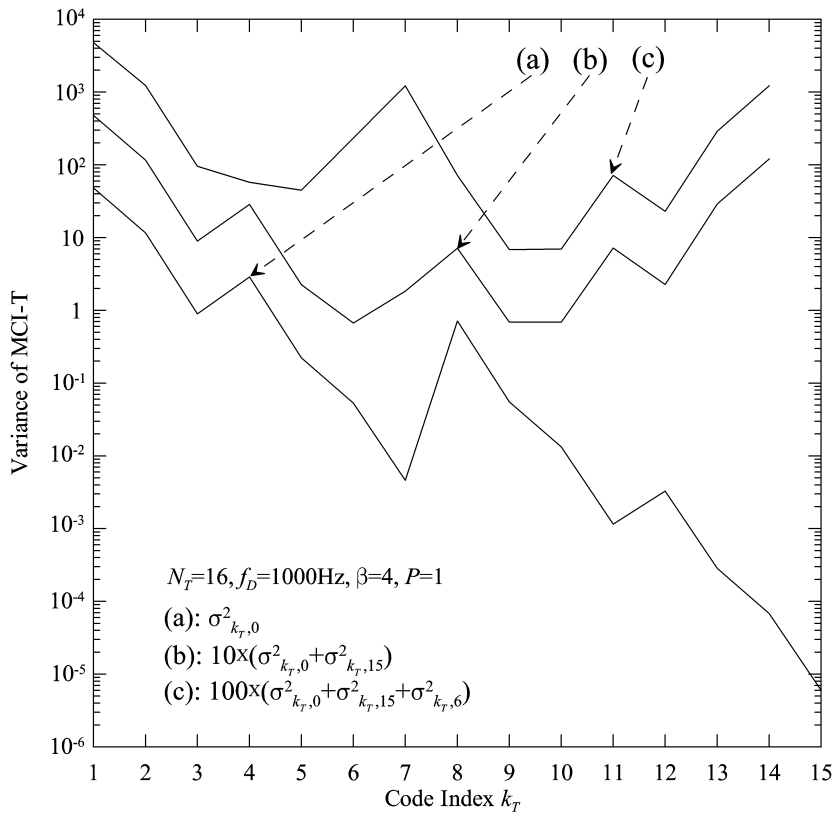

Fig. 4. Variance of MCI-T without weighting in time domain.

MMSE weights in frequency domain. The MCI-T caused by the pilot channel with code $C_{N_{T}}^{(0)}$ to the data channel with code $C_{N_{T}}^{\left(k_{T}\right)}$ is given by

$$
I_{k_{T}, 0}^{(T)}=\sqrt{\beta P} d_{P}\left(\sum_{\bar{i}=0}^{N_{T}-1} \lambda_{\bar{m}, i} c_{N_{T}, \bar{i}}^{\left(k_{T}\right)}\right)
$$

whereas the MCI-T caused by the data channel with code $C_{N_{T}}^{\left(\bar{k}_{T}\right)}$ is given by

$$
I_{k_{T}, \bar{k}_{T}}^{(T)}=\sqrt{P} d_{k}\left(\sum_{\bar{i}=0}^{N_{T}-1} \lambda_{\bar{m}, \bar{i}} c_{N_{T}, \bar{i}}^{\left(k_{T}\right)} c_{N_{T}, \bar{i}}^{\left(\bar{k}_{T}\right)}\right)
$$

Assuming that the channel is fixed within one effective OFCDM time chip duration, i.e., $\lambda_{\bar{m}, \bar{i}} \approx H_{\bar{m}}(\bar{i} T)$, the variances of $I_{k_{T}, 0}^{(T)}$ and $I_{k_{T}, \bar{k}_{T}}^{(T)}$ are given by

$$
\sigma_{k_{T}, 0}^{2}=\beta P\left(\sum_{i_{0}=0}^{N_{T}-1} \sum_{i_{1}=0}^{N_{T}-1} \rho_{t}\left(i_{0}, i_{1}\right) c_{N_{T}, i_{0}}^{\left(k_{T}\right)} c_{N_{T}, i_{1}}^{\left(k_{T}\right)}\right)
$$

and

$$
\sigma_{k_{T}, \bar{k}_{T}}^{2}=P\left[\sum_{i_{0}=0}^{N_{T}-1} \sum_{i_{1}=0}^{N_{T}-1}\left(\begin{array}{l}
\rho_{t}\left(i_{0}, i_{1}\right) c_{N_{T}, i_{0}}^{\left(k_{T}\right)} c_{N_{T}, i_{1}}^{\left(k_{T}\right)} \\
c_{N_{T}, i_{0}}^{\left(\bar{k}_{T}\right)} c_{N_{T}, i_{1}}^{\left(\bar{k}_{T}\right)}
\end{array}\right)\right],
$$

respectively. Since in a non-static fading channel (i.e., with non-zero Doppler shift), the time correlation $\rho_{t}\left(i_{0}, i_{1}\right)$ is a function of $\left|i_{0}-i_{1}\right|$, the variances are related to $C_{N_{T}}^{\left(k_{T}\right)}$ and $C_{N_{T}}^{\left(\bar{k}_{T}\right)}$. First of all, consider the variance $\sigma_{k_{T}, 0}^{2}$. Given $N_{T}=$ $16, \beta=0.25 \times 16=4, P=1$, and $f_{D}=1000 \mathrm{~Hz}, \sigma_{k_{T}, 0}^{2}$ is plotted as (a) in Fig. 4 as a function of the code index $k_{T}$. After the pilot code, the second code to be assigned in time domain should be the one suffering from the smallest MCI-T caused by $C_{N_{T}}^{(0)}$. Apparently, it is $C_{16}^{(15)}$. In order to assign a third code, the variances, $\sigma_{k_{T}, 15}^{2}$, of MCI-T caused by $C_{16}^{(15)}$ to all other codes are computed. The scaled sum of the two variances, $\sigma_{k_{T}, 0}^{2}+\sigma_{k_{T}, 15}^{2}$, is shown as (b) in Fig. 4. It can be seen that $C_{16}^{(6)}$ suffers from the smallest sum and therefore, it is the third code to be assigned. The fourth code can be assigned by selecting the code which suffers from the smallest sum $\sigma_{k_{T}, 0}^{2}+\sigma_{k_{T}, 15}^{2}+\sigma_{k_{T}, 6}^{2}$ as (c) in Fig. 4. It can be seen that $C_{16}^{(9)}$ is the fourth code to be assigned. Similar procedures can be kept going on until the orders of all codes are assigned.

The code assignment schemes obtained by minimizing MCI-T can be interpreted using code distances defined in Section II, since MCIs are dependant on the code distance. The smaller the code distance, the larger the MCIs. As shown in Fig. 1, the code distance between the pilot code $C_{N_{T}}^{(0)}$ and $C_{N_{T}}^{(1)}$ is one while the distance between $C_{N_{T}}^{(0)}$ and $C_{N_{T}}^{\left(N_{T}-1\right)}$ is $N_{T} / 2$. According to (16), the interferences caused by the pilot channel to the data channels with codes $C_{N_{T}}^{(1)}=\{1, \cdots, 1,-1, \cdots,-1\}$ and $C_{N_{T}}^{\left(N_{T}-1\right)}=$ $\{1,-1,-1,1,-1,1,1,-1, \cdots\}$ are given by

$$
\begin{aligned}
I_{1,0}^{(T)} & =\sqrt{\beta P} d_{P}\left(\sum_{\bar{i}=0}^{N_{T}-1} \lambda_{\bar{m}, i} c_{N_{T}, \bar{i}}^{(1)}\right) \\
& =\sqrt{\beta P} d_{P}\left[\begin{array}{l}
\lambda_{\bar{m}, 0} \cdot 1+\cdots+\lambda_{\bar{m}, N_{T} / 2-1} \cdot 1 \\
+\lambda_{\bar{m}, N_{T} / 2} \cdot(-1)+\cdots+\lambda_{\bar{m}, N_{T}-1} \cdot(-1)
\end{array}\right] \\
& =\sqrt{\beta P} d_{P} \sum_{\bar{i}=0}^{N_{T} / 2-1}\left(\lambda_{\bar{m}, \bar{i}}-\lambda_{\bar{m}, \bar{i}+N_{T} / 2}\right)
\end{aligned}
$$

and

$$
\begin{aligned}
I_{N_{T}-1,0}^{(T)} & =\sqrt{\beta P} d_{P}\left(\sum_{\bar{i}=0}^{N_{T}-1} \lambda_{\bar{m}, \bar{i}} c_{N_{T}, \bar{i}}^{\left(N_{T}-1\right)}\right) \\
& =\sqrt{\beta P} d_{P}\left[\begin{array}{l}
\lambda_{\bar{m}, 0} \cdot 1+\lambda_{\bar{m}, 1} \cdot(-1) \\
+\lambda_{\bar{m}, 2} \cdot(-1)+\lambda_{\bar{m}, 3} \cdot(1)+\cdots
\end{array}\right] \\
& =\sqrt{\beta P} d_{P} \sum_{\bar{i}=0}^{N_{T} / 2-1}(-1)^{\delta(\bar{i})}\left(\lambda_{\bar{m}, 2 \bar{i}}-\lambda_{\bar{m}, 2 \bar{i}+1}\right),
\end{aligned}
$$

respectively, where

$$
\delta(\bar{i})= \begin{cases}0, & \bar{i}=0,3,5,6, \cdots \\ 1, & \bar{i}=1,2,4,7, \cdots\end{cases}
$$

The correlation of the fading factors $\lambda_{\bar{m}, \bar{i}}$ and $\lambda_{\bar{m}, \bar{j}}$ should be inversely proportional to the difference $|\bar{i}-\bar{j}|$ of the time index. The differences in the time index between $\lambda_{\bar{m}, 2 \bar{i}+1}$ and $\lambda_{\bar{m}, 2 \bar{i}}$ and between $\lambda_{\bar{m}, \bar{i}}$ and $\lambda_{\bar{m}, \bar{i}+N_{T} / 2}$ are one and $N_{T} / 2$, respectively. Therefore, when $N_{T}>2$, the correlation between $\lambda_{\bar{m}, 2 \bar{i}}$ and $\lambda_{\bar{m}, 2 \bar{i}+1}$ should be larger than that between $\lambda_{\bar{m}, \bar{i}}$ and $\lambda_{\bar{m}, \bar{i}+N_{T} / 2}$, so that $I_{1,0}^{(T)}$ should be greater than $I_{N_{T}-1,0}^{(T)}$. In order to make the MCI-T small, the code $C_{N_{T}}^{\left(N_{T}-1\right)}$ should be assigned with higher priority than $C_{N_{T}}^{(1)}$, which is consistent with the code assignment schemes obtained by minimizing MCI-T.

In time domain code assignment, special consideration should be taken on the MCI-T caused by the pilot channel since it has a higher power than that on a data channel. From 
TABLE I

Non-Sequential Code Assignment Schemes

Time Domain Spreading Codes $\left(C_{N_{T}}^{(1)}\right.$ is not assigned when $\left.N_{T}=16\right)$

\begin{tabular}{|c|c|c|c|c|c|c|c|c|c|c|c|c|c|c|c|}
\hline $\begin{array}{c}\text { Assignment } \\
\text { order }\end{array}$ & $\begin{array}{c}1 \text { (For } \\
\text { pilot) }\end{array}$ & 2 & 3 & 4 & 5 & 6 & 7 & 8 & 9 & 10 & 11 & 12 & 13 & 14 & 15 \\
\hline$N_{T}=4$ & $C_{4}^{(0)}$ & $C_{4}^{(3)}$ & $C_{4}^{(2)}$ & $C_{4}^{(1)}$ & & & & & & & & & & & \\
\hline$N_{T}=8$ & $C_{8}^{(0)}$ & $C_{8}^{(7)}$ & $C_{8}^{(3)}$ & $C_{8}^{(4)}$ & $C_{8}^{(6)}$ & $C_{8}^{(5)}$ & $C_{8}^{(2)}$ & $C_{8}^{(1)}$ & & & & & & & \\
\hline$N_{T}=16$ & $C_{16}^{(0)}$ & $C_{16}^{(15)}$ & $C_{16}^{(6)}$ & $C_{16}^{(9)}$ & $C_{16}^{(12)}$ & $C_{16}^{(10)}$ & $C_{16}^{(5)}$ & $C_{16}^{(3)}$ & $C_{16}^{(14)}$ & $C_{16}^{(7)}$ & $C_{16}^{(11)}$ & $C_{16}^{(13)}$ & $C_{16}^{(8)}$ & $C_{16}^{(4)}$ & $C_{16}^{(2)}$ \\
\hline
\end{tabular}

Frequency Domain Spreading Codes

\begin{tabular}{|c|c|c|c|c|c|c|c|c|c|c|c|c|c|c|c|c|}
\hline $\begin{array}{c}\text { Assignment } \\
\text { order }\end{array}$ & 1 & 2 & 3 & 4 & 5 & 6 & 7 & 8 & 9 & 10 & 11 & 12 & 13 & 14 & 15 & 16 \\
\hline$N_{T}=4$ & $C_{4}^{(0)}$ & $C_{4}^{(2)}$ & $C_{4}^{(3)}$ & $C_{4}^{(1)}$ & & & & & & & & & & & & \\
\hline$N_{T}=8$ & $C_{8}^{(0)}$ & $C_{8}^{(4)}$ & $C_{8}^{(6)}$ & $C_{8}^{(2)}$ & $C_{8}^{(5)}$ & $C_{8}^{(3)}$ & $C_{8}^{(7)}$ & $C_{8}^{(1)}$ & & & & & & & & \\
\hline$N_{T}=16$ & $C_{16}^{(0)}$ & $C_{16}^{(8)}$ & $C_{16}^{(12)}$ & $C_{16}^{(4)}$ & $C_{16}^{(10)}$ & $C_{16}^{(6)}$ & $C_{16}^{(14)}$ & $C_{16}^{(2)}$ & $C_{16}^{(9)}$ & $C_{16}^{(5)}$ & $C_{16}^{(13)}$ & $C_{16}^{(3)}$ & $C_{16}^{(11)}$ & $C_{16}^{(7)}$ & $C_{16}^{(15)}$ & $C_{16}^{(1)}$ \\
\hline
\end{tabular}

the curve (a) in Fig. 4, it can be seen that $C_{N_{T}}^{(1)}$ suffers from much more interference from the pilot than other codes due to the shorter code distance between $C_{N_{T}}^{(1)}$ and $C_{N_{T}}^{(0)}$. In the curve (b), the interference on $C_{N_{T}}^{(14)}$ becomes large, due to that after the code $C_{N_{T}}^{(15)}$ has been assigned, it causes large MCI-T to $C_{N_{T}}^{(14)}$ because the code distance between $C_{N_{T}}^{(14)}$ and $C_{N_{T}}^{(15)}$ is only one. Similarly, in the curve (c), the interference on $C_{N_{T}}^{(7)}$ is large since the adjacent code, $C_{N_{T}}^{(6)}$, has been assigned. Further, the variance of the interference on $C_{N_{T}}^{(1)}$ is still about three times larger than that on $C_{N_{T}}^{(7)}$ and $C_{N_{T}}^{(14)}$ due to the fact that the power on the pilot channel is four times as large as that on one data channel, i.e., $\beta=4$. Note that $\beta$ increases with the number of data channels $K$ and could be much larger than four in an OFCDM system with 2-D spreading. In that case, the interference caused by the pilot channel is even more serious. Thus, the code $C_{N_{T}}^{(1)}$, adjacent to the pilot code, is not suitable to be assigned. Moreover, the MCI-T also depends on the channel variation in time domain, or the Doppler shifts. Given the same Doppler shift, the channel variation in $N_{T}$ OFCDM symbol duration increases as $N_{T}$ increases, which results in a higher MCI-T for a larger $N_{T}$. In summary, in order to avoid large pilot interference, $C_{N_{T}}^{(1)}$ is suggested not be used for data transmission when $N_{T}$ is large, although it can be used when $N_{T}$ is small (i.e., $N_{T}=4$ or 8 ). The code assignment for $N_{T}=4$ and 8 can be carried out as for $N_{T}=16$. The complete non-sequential code assignment schemes in time domain are shown in Table I for $N_{T}$ equal to 4,8 , and 16 , respectively.

\section{B. Frequency Domain Code Assignment}

One difference between time domain and frequency domain in code assignment is that the code $C_{N_{T}}^{(0)}$ is used for pilot in time domain, whereas there is no need to have a pilot code in frequency domain. It can be seen from (15) that the residual MCI-F is the original MCI-F (first term on the right hand side of (15) with $s=0)$ subtracted by the regenerated MCI-F. Although the hybrid detection can cancel out the MCI-F much, good code assignment helps mitigate the original MCI-F further. The original MCI-F is caused by the data channels in the code set $\Omega_{F}$ and $\lambda_{\bar{m}, \bar{i}}$. Assuming $w_{f, \bar{m}}^{(0)} \equiv 1, w_{t, \bar{m}, \bar{i}} \equiv 1$ for all $\bar{m}$ and $\lambda_{\bar{m}, \bar{i}}$ to be unchanged for all $\vec{i}$, the MCI-F caused by the channel with code $C_{N_{F}}^{\left(\bar{k}_{F}\right)}$ to the channel with code $C_{N_{F}}^{\left(k_{F}\right)}$ is expressed as

$$
I_{k_{F}, \bar{k}_{F}}^{(F)}=\sqrt{P} d_{\bar{k}} N_{T}\left(\sum_{\bar{m}=0}^{N_{F}-1} \lambda_{\bar{m}, \bar{i}} c_{N_{F}, \bar{m}}^{\left(k_{F}\right)} c_{N_{F}, \bar{m}}^{\left(\bar{k}_{F}\right)}\right)
$$

with variance

$\sigma_{k_{F}, \bar{k}_{F}}^{2}=P \cdot N_{T}^{2}\left[\sum_{m_{0}=0}^{N_{F}-1} \sum_{m_{1}=0}^{N_{F}-1}\left(\begin{array}{c}\rho_{f}\left(m_{0}, m_{1}\right) c_{N_{F}, m_{0}}^{\left(k_{F}\right)} \\ c_{N_{F}, m_{1}}^{\left(k_{F}\right)} c_{N_{F}, m_{0}}^{\left(\bar{k}_{F}\right)} c_{N_{F}, m_{1}}^{\left(\bar{k}_{F}\right)}\end{array}\right)\right]$

Similar to (17) and (19), since in the broadband channel, the frequency correlation $\rho_{f}\left(m_{0}, m_{1}\right)$ is a function of $\left|m_{0}-m_{1}\right|$, the variance depends on $C_{N_{F}}^{\left(\bar{k}_{F}\right)}$ and $C_{N_{F}}^{\left(k_{F}\right)}$. Assuming $\bar{k}_{F}=0$ (i.e., $C_{N_{F}}^{(0)}$ is assigned first), consider the variances $\sigma_{k_{F}, 0}^{2}$. With $N_{T}=1, N_{F}=16, P=1$ and $\Delta f_{c}=2.5 \mathrm{MHz}$ $\left(\tau_{r m s} \approx 0.4 \mu \mathrm{s}\right), \sigma_{k_{F}, 0}^{2}$ is shown as (a) in Fig. 5 as a function of the code index $k_{F}$. After $C_{N_{F}}^{(0)}$, the second code to be assigned in frequency domain should be the one suffering from the smallest MCI-F caused by $C_{N_{F}}^{(0)}$. That is $C_{16}^{(8)}$. In order to assign a third code, the variances, $\sigma_{k_{F}, 8}^{2}$, of MCIF caused by $C_{16}^{(8)}$ to all other codes are computed. The sum of the two variances, $\sigma_{k_{F}, 0}^{2}+\sigma_{k_{F}, 8}^{2}$, is shown as (b) in Fig. 5. It can be seen that $C_{16}^{(12)}$ suffers from the smallest sum and therefore, it is the third code to be assigned. The fourth code can be assigned by selecting the code which suffers from the smallest sum $\sigma_{k_{F}, 0}^{2}+\sigma_{k_{F}, 8}^{2}+\sigma_{k_{F}, 12}^{2}$ as (c) in Fig. 5. It can be seen that $C_{16}^{(4)}$ is the fourth code to be assigned. Similar procedures can be kept going on until the orders of all codes are assigned. The code assignment for $N_{F}=4$ and 8 can be carried out as for $N_{F}=16$. The complete non-sequential code assignment in frequency domain is shown in Table I. It can be seen from the table that the code assignment schemes are different in time and frequency 


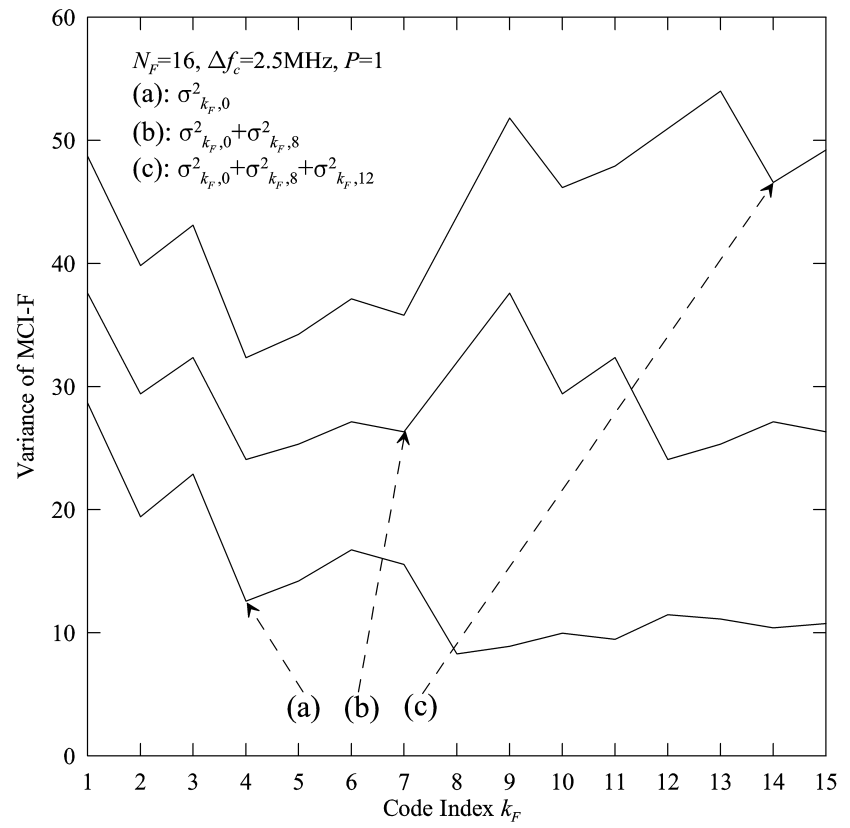

Fig. 5. Variance of MCI-F without weighting in frequency domain.

domains since the channel correlation properties are different. However, the code assignment schemes in frequency domain can also be interpreted by the code distance. The codes with larger distances to each other should be assigned with higher priority while those with shorter distances should be used with lower priority.

\section{2-D Code Assignment}

As discussed before, the MCIs are caused by multi-code channels and channel variations/selectivity. Since the broadband channel is highly frequency selective, the MCI-F caused by codes in $\Omega_{F}$ should be more serious than the MCI-T caused by codes in $\Omega_{T}$, even in the case of a large Doppler shift. This is because the channel variation over $N_{T}$ consecutive OFCDM time chips in time domain is much less than that over $N_{F}$ interleaved symbols in frequency domain. Moreover, since the powerful pilot can cause much interference to the adjacent code $C_{N_{T}}^{(1)}$, this code is not suggested be used when $N_{T}=16$. Thus it is anticipated that the overall interference caused in frequency domain should be more than that caused in time domain. In order to verify this, Fig. 6 shows the variances of the MCI-T and residual MCI-F (see (14) and (15)) as a function of Doppler shifts $f_{D}$. It is assumed that $N=16 \times 16$, $S N R_{b}=16 \mathrm{~dB}$ and $\Delta f_{c}=2.5 \mathrm{MHz}\left(\tau_{r m s} \approx 0.4 \mu \mathrm{s}\right)$. Twostage hybrid detection is used. If the number of data codes is $K=N_{T}-2=14$ and 14 time domain spreading codes are employed with only one frequency domain spreading code $C_{16}^{(0)}, 14$ 2-D codes $\left\{C_{16}^{\left(k_{T}\right)}, C_{16}^{(0)}\right\}\left(k_{T}=2, \cdots, 15\right)$ are used. Thus, there is only MCI-T on the data channels but no MCI-F. Conditional variances of MCI-Ts are calculated for individual data channels first. Then the variance is obtained by averaging the conditional variances over all data channels. On the other hand, if the number of data codes is $K=N_{F}=16$, and 16 frequency domain spreading codes are used with only one time domain code $C_{16}^{(15)}, 162$-D codes $\left\{C_{16}^{(15)}, C_{16}^{\left(k_{F}\right)}\right\}$ $\left(k_{F}=0, \cdots, 15\right)$ are used. It can be seen from Fig. 6 that the

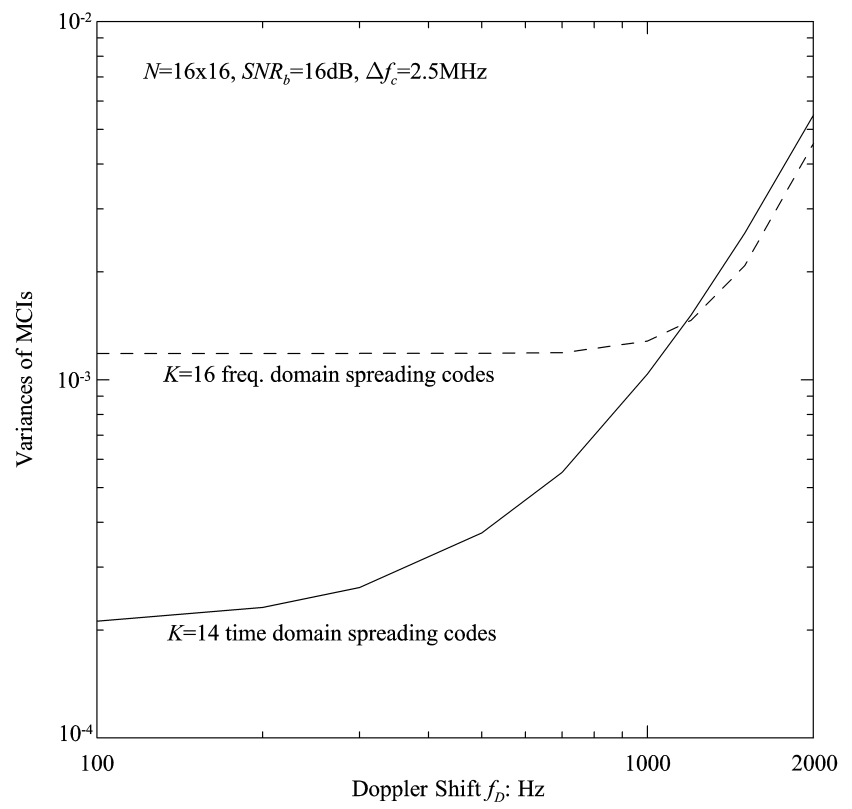

Fig. 6. Comparison of MCI-T and MCI-F.

variance of MCI-F is much larger than that of MCI-T when the Doppler shift is less than $1000 \mathrm{~Hz}$. Thus, the code assignment in time domain should be carried out first. That is, a frequency domain spreading code $C_{N_{F}}^{(0)}$ is chosen firstly, and up to $N_{T}-1$ (or $N_{T}-2$ if $N_{T}=16$ ) different time domain spreading codes are assigned. When more code channels are needed, other frequency domain spreading codes will be assigned.

\section{Numerical Results}

Given the decision variable $y_{\frac{1}{k}}^{(s)}$ in (11), the bit error rate (BER) performance of the OFCDM system can be evaluated by a semi-analytical approach [18-19]. The basic idea is firstly to calculate the value of the useful signal component $S_{\bar{k}}^{(s)}$ and MCI-F term, $M C I_{\bar{k}}^{(F)}(s)$, given data symbols and channel information. Then the MCI-T, $M C I_{\bar{k}}^{(T)}(s)$, can be modeled as an additive Gaussian noise and the conditional BER can be obtained. This conditional BER is firstly averaged over all possible data symbols involved in $M C I_{\bar{k}}^{(F)}(s)$, then averaged over all channel information by using a Monte Carlo simulation [22]. Using the semi-analytical approach, numerical results can be obtained to evaluate the system performance. The performance of the proposed 2-D nonsequential code assignment scheme is investigated with various channel conditions. The BERs are obtained firstly for individual channel codes and then the average BER is obtained by averaging the individual BERs over all employed data channels. The worst BER is computed on the code which has the highest BER. For comparison, the performance of a simple sequential code assignment scheme is also shown. Without any special design criterion, this scheme assigns 2-D codes sequentially according to their code indices starting with $\left\{C_{N_{T}}^{(0)}, C_{N_{F}}^{(0)}\right\}$, then $\left\{C_{N_{T}}^{(1)}, C_{N_{F}}^{(0)}\right\}, \ldots$, and $\left\{C_{N_{T}}^{\left(N_{T}-1\right)}, C_{N_{F}}^{(0)}\right\}$. When more codes are needed, $C_{N_{F}}^{(1)}$ replaces $C_{N_{F}}^{(0)}$, combined with $C_{N_{T}}^{(1)} \sim C_{N_{T}}^{\left(N_{T}-1\right)}$, and so on.

In order to show the performance of the proposed nonsequential code assignment scheme in a fast fading (i.e., 


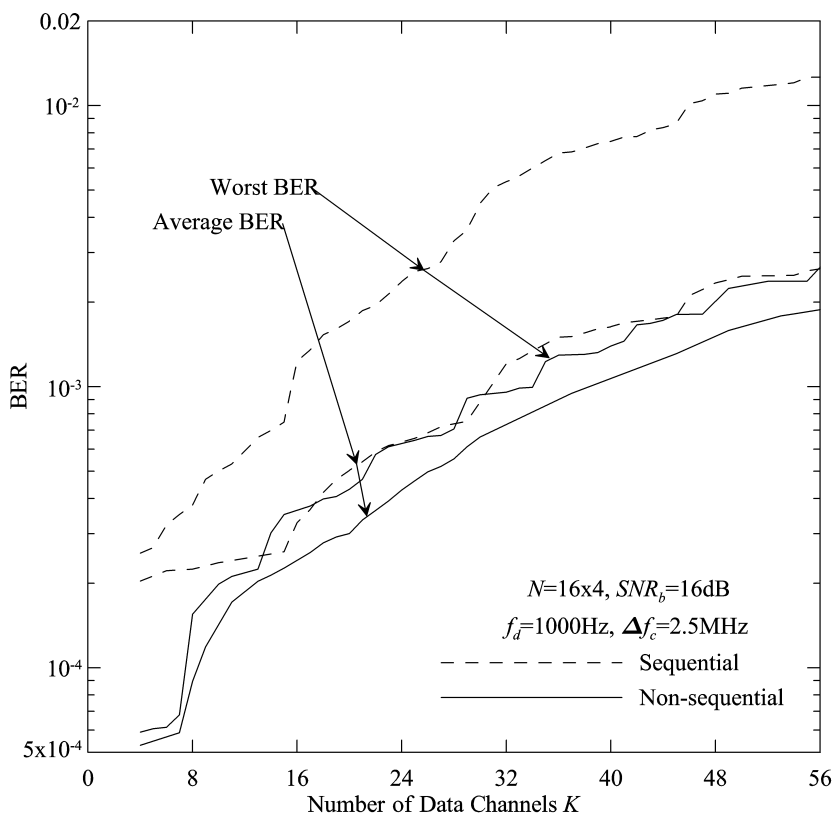

Fig. 7. Performance of code assignment schemes as a function of $K$ in fast fading.

$\left.f_{D}=1000 \mathrm{~Hz}\right)$ and highly frequency selective channel (i.e., $\Delta f_{c}=2.5 \mathrm{MHz}$ or $\tau_{r m s} \approx 0.4 \mu \mathrm{s}$ ), both the worst and average BERs of 2-stage hybrid detection are plotted in Fig. 7 as a function of the number, $K$, of data channels. Given $N=16 \times 4$, according to the non-sequential assignment schemes, the time domain spreading code $C_{N_{T}}^{(1)}$ is not used although it is used in the sequential scheme. First of all, it can be seen that both the average and the worst BER performances degrade when $K$ increases. Note that each data channel suffers from interference from other $K-1$ data channels and the pilot channel. When $K$ increases, more interference is caused by other data channels. Moreover, the power on the pilot channel also increases with $K$ due to $\beta=0.25 K$. As a result, each data channel experiences more interference as $K$ gets larger and both the average and worst BER increase. Moreover, it can be seen that the BERs increases with $K$ in a manner like step, especially the worst BERs. This is because the variance of the MCI depends on which codes to be used. If a code caused high MCI is assigned, the BER increases rapidly, while if a code resulting in little MCI is used, the BER is almost flat. Using sequential scheme, a rapid increase in BER occurs every $N_{T}-1=15$ code channels. According to the sequential scheme, the first $N_{T}-1$ code channels use the same frequency domain spreading code $C_{N_{F}}^{(0)}$ and different time domain spreading code. Hence, there is only MCI-T and no MCI-F. As $K$ is increased to $N_{T}$, a new frequency domain spreading code $C_{N_{F}}^{(1)}$ should be used, thus MCI-F occurs. The resultant total MCI is much larger than the MCI when $K=N_{T}-1$. So the BER performance degrades suddenly every $N_{T}-1$ code channels. On the other hand, for the non-sequential scheme, a step occurs in the worst BER every $N_{T}-2=14$ code channels since only $N_{T}-2$ time domain spreading codes are considered for data transmission. Moreover, there is another step in the middle of the $N_{T}-2$ code channels. Referring to Table I, it can be seen that the first $N_{T} / 2-1=7$ assigned codes have large code distance to each other, so the MCI-T between these code channels are relatively small. But the $8^{\text {th }}$ assigned code $C_{N_{T}}^{(14)}$ has a small code distance of one to the firstly assigned code $C_{N_{T}}^{(15)}$, which results in a much higher MCI-T between these two code channels compared to the MCI-T before the $8^{\text {th }}$ code is assigned. Hence, the worst BER becomes large suddenly. Furthermore, from the average BER, it can be seen that the non-sequential scheme provides better performance than the sequential scheme, especially at low to medium system loads (or small $K$ ). The most significant improvement in the average BER is obtained for $K \leq 14$, then the improvement decreases as $K$ increases. On the other hand, a bigger difference in the worst BERs can be viewed. Although it cannot be shown in the figure, in the sequential scheme, the worst BER occurs on the data channels with time domain spreading code $C_{N_{T}}^{(1)}$ due to the large MCI-T caused by the pilot channel. Since $C_{N_{T}}^{(1)}$ is not used in the non-sequential scheme, there is less interference from the powerful pilot channel, so that the worst BER is much less than that of the sequential one. For the non-sequential scheme, the worst BER and the average BER are close to each other. That is, different data channels present similar BERs, which is desirable. On the contrary, in the sequential scheme, the worst BER is much larger than that of the average BER. That is, the data channel with the time domain spreading code $C_{N_{T}}^{(1)}$ contains errors more likely than other data channels. In conclusion, for a large range of the system load, the nonsequential assignment scheme outperforms the sequential one.

Given $N=16 \times 4, K=20$ (or $33.3 \%$ load), and $\Delta f_{c}=2.5 \mathrm{MHz}\left(\tau_{r m s} \approx 0.4 \mu \mathrm{s}\right)$, the system performance is shown in Fig. 8 as a function of $S N R_{b}$. Again, the 2-stage hybrid detection is considered. The system performance is evaluated with $f_{D}=500 \mathrm{~Hz}$ in Fig. 8(a) and $f_{D}=1000 \mathrm{~Hz}$ in Fig. 8(b). It can be seen from both figures that with either the non-sequential or sequential code assignment scheme, both the average and worst BERs improve as $S N R_{b}$ increases and the performance gap between the two schemes increases with $S N R_{b}$. The reason is that the system performance depends on both $S N R_{b}$ and MCI. When $S N R_{b}$ becomes larger, the influence of background noise reduces and the MCI plays a more and more important role in determining the system performance. Since the non-sequential scheme results in a much smaller MCI in the system, its advantage over the sequential scheme is more obvious as $S N R_{b}$ increases. Moreover, given $S N R_{b}$, the performance gap between the two schemes is smaller with $f_{D}=500 \mathrm{~Hz}$ than that with $f_{D}=1000 \mathrm{~Hz}$, since the system suffers from less MCI with a smaller Doppler shift. It can also be seen that with $f_{D}=500 \mathrm{~Hz}$, the BERs improve rapidly as $S N R_{b}$ increases, and presents no obvious error floor for both non-sequential and sequential schemes. However, in case of $f_{D}=1000 \mathrm{~Hz}$, the BERs improvement becomes smaller when $S N R_{b}$ is higher, and there is an error floor in the worst BER of sequential scheme. Apparently, the system becomes MCI-limited at high $S N R_{b}$. Since the nonsequential scheme can effectively reduce the MCI, the error floor is much less serious than that in the sequential scheme. In summary, for both $f_{D}=500 \mathrm{~Hz}$ and $f_{D}=1000 \mathrm{~Hz}$, the non-sequential assignment scheme outperforms the sequential scheme, especially at middle to high $S N R_{b}$. 


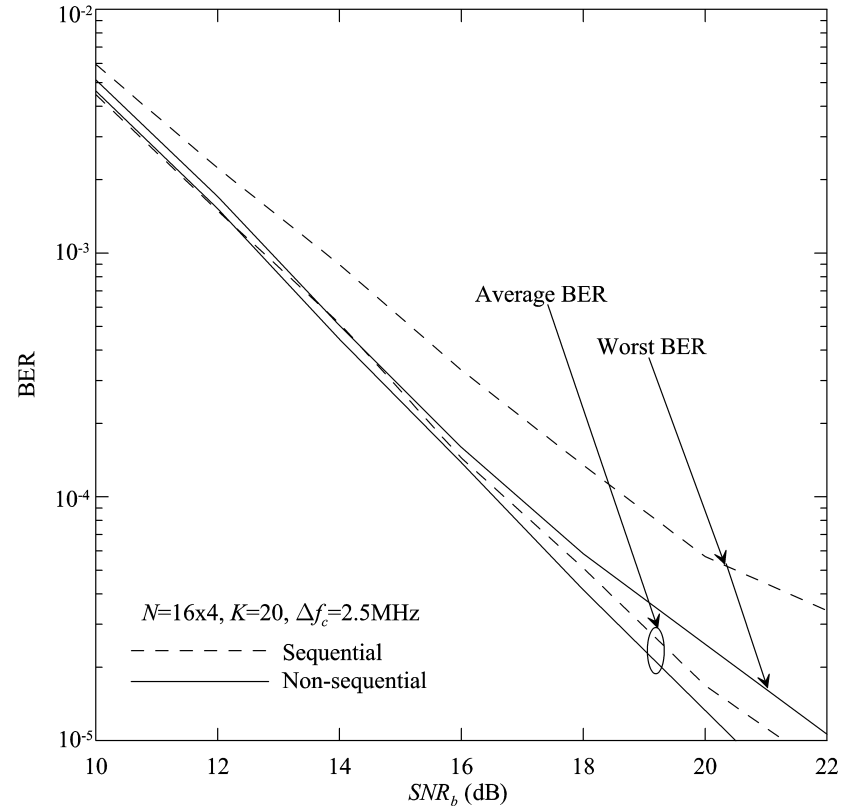

(a) $f_{D}=500 \mathrm{~Hz}$

Fig. 8. System performance as a function of $S N R_{b}$.

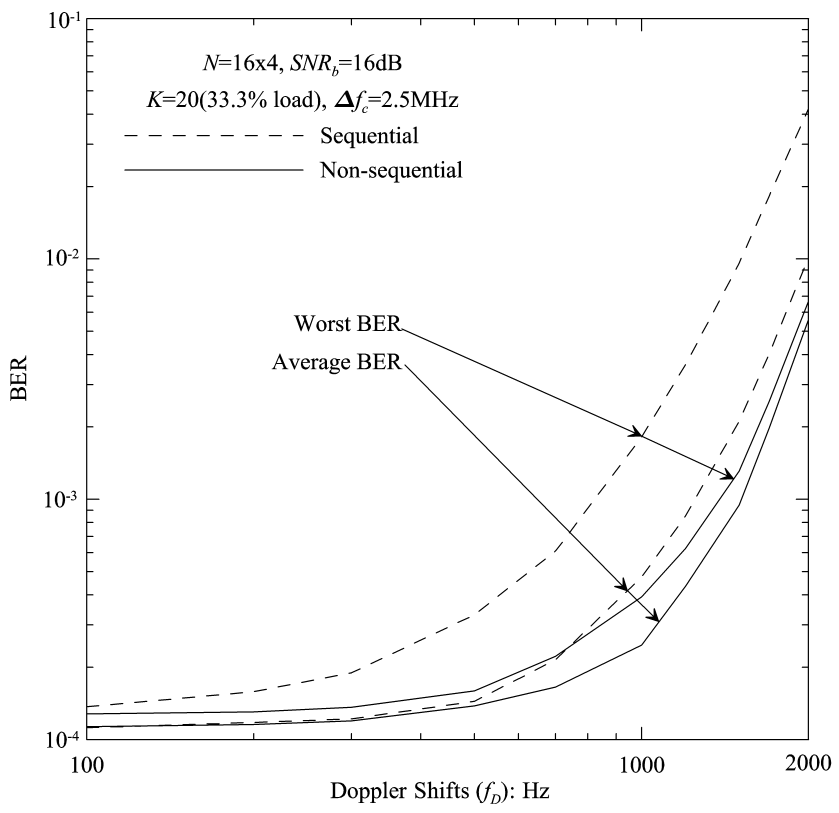

Fig. 9. Effect of Doppler shift.

Finally, Fig. 9 illustrates the effect of Doppler shift, $f_{D}$, on the performance of the non-sequential and sequential code assignment schemes, when $S N R_{b}=16 \mathrm{~dB}, N=16 \times 4$, $K=20$ (or $33.3 \%$ load) and $\Delta f_{c}=2.5 \mathrm{MHz}\left(\tau_{r m s} \approx 0.4 \mu \mathrm{s}\right)$. Both the average and worst BERs of the 2-stage hybrid detection are plotted. It can be seen that both the worst and average BERs of the two code assignment schemes increase as the Doppler shift increases, due to the increasing MCI-T and deteriorating channel estimation. Although the average BERs of the two assignment schemes are close, the worst BER of the non-sequential assignment is much smaller than that of the sequential assignment, especially when the Doppler is not small. This is mainly due to that the code $C_{N_{T}}^{(1)}$ is

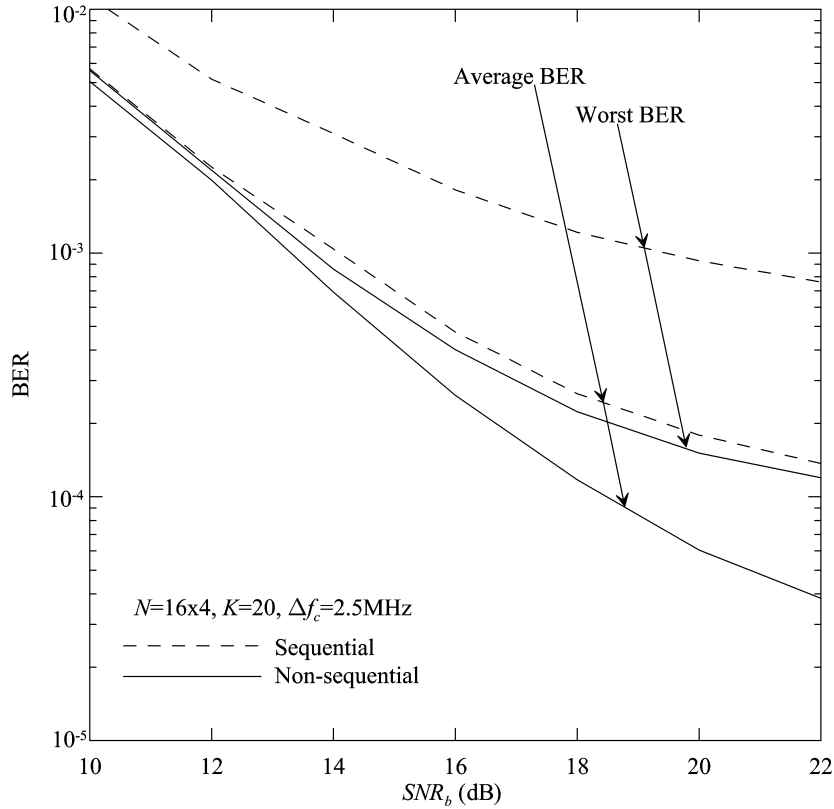

(b) $f_{D}=1000 \mathrm{~Hz}$

not used in the non-sequential scheme, whereas it is used in the sequential scheme. When the Doppler shift is small, the performance difference between both assignment schemes is very small because of the decreasing interference caused to the $C_{N_{T}}^{(1)}$ channel by the pilot. Furthermore, the sequential scheme performs very similarly to the non-sequential scheme when the Doppler is very small. In summary, for a large range of the Doppler shift, the non-sequential scheme outperforms the sequential scheme.

\section{CONCLUSION}

In order to mitigate MCI, non-sequential code assignment schemes have been proposed in OFCDM systems. The following conclusions are drawn:

1) Code assignment schemes are proposed aiming to mitigate MCI in OFCDM systems and can be interpreted by using the code distance defined for OVSF codes. The smaller the code distance, the bigger the MCI is caused to each other. The codes with larger code distances to each other should be assigned with higher priority;

2) When $N_{T}$ is large, i.e., 16 , the time domain spreading code adjacent to the pilot code, $C_{N_{T}}^{(1)}$, is not suggested be used. Moreover, time domain spreading codes should be assigned with higher priorities than frequency domain spreading codes;

3) For a large range of the system load or a large range of the Doppler shift, the non-sequential assignment scheme outperforms the sequential scheme, especially at middle to high $S N R_{b}$.

\section{ACKNOWLEDGMENT}

This work was supported by The University of Hong Kong Research Committee. The authors would like to thank Dr. M. Sawahashi, Dr. K. Higuchi, Dr. H. Atarashi, and Dr. N. Maeda from NTT DoCoMo, Japan, for their early helpful discussions. 


\section{REFERENCES}

[1] T. Ikeda, K. Okawa, M. Sawahashi, and F. Adachi, "Experimental evaluation of coherent rake combining for broadband single-carrier DSCDMA wireless communications," IEICE Trans. Commun., vol. E84-B, no. 3, pp. 415-424, Mar. 2001.

[2] J. Chuang and N. Sollenberger, "Beyond 3G: wideband wireless data access based on OFDM and dynamic packet assignment," IEEE Commun. Mag., vol. 38, no. 7, pp. 78-87, July 2000.

[3] H. Atarashi, S. Abeta, and M. Sawahashi, "Broadband packet wireless access appropriate for high-speed and high-capacity throughput," in Proc. IEEE VTC2001-Spring, pp. 566-570, May 2001.

[4] A. Persson, T. Ottosson, and E. Strom, "Time-frequency localized CDMA for downlink multi-carrier systems," in Proc. IEEE ISSSTA 2002, pp. 118122, Sept. 2002.

[5] A. Matsumoto, K. Miyoshi, M. Uesugi, and O. Kato, "A study on time domain spreading for OFCDM," in Proc. 5th International Symposium on Wireless Personal Multimedia Communications, vol. 2, pp. 725-728, Oct. 2002.

[6] N. Maeda, Y. Kishiyama, and M. Sawahashi, "Variable spreading factorOFCDM with two dimensional spreading that prioritizes time domain spreading for forward link broadband wireless access," in Proc. IEEE VTC2003-spring, pp. 127-132, Apr. 2003.

[7] Chih-Lin I and R. D. Gitlin, "Multi-code CDMA wireless personal communications networks," in Proc. IEEE ICC'95, pp. 1060-1064, June 1995.

[8] E. Dahlman, B. Gudmundson, M. Nilsson, and J. Skold, "UMTS/IMT2000 based on wideband CDMA," IEEE Commun. Mag., vol. 36, pp. 7080, Sept. 1998.

[9] F. Adachi, M. Sawahashi, and H. Suda, "Wideband DS-CDMA for next-generation mobile communications systems," IEEE Commun. Mag., vol. 36 , no. 9 , pp. 56-69, Sept. 1998.

[10] T. Minn and K. Y. Siu, "Dynamic assignment of orthogonal variablespreading-factor codes in W-CDMA," IEEE J. Select. Areas Commun., vol. 18, no. 8, pp. 1429-1440, Aug. 2000.

[11] C. M. Chao, Y. C. Tseng, and L. C. Wan, "Reducing internal and external fragmentations of OVSF codes in WCDMA systems with multiple codes," Wireless Commun. and Networking, vol. 1, pp. 693-698, Mar. 2003.

[12] P. J. Wan, X. Y. Li, and O. Frieder, "OVSF-CDMA code assignment in wireless ad hoc networks," in Proc. DIALM-POMC 2004, pp. 92-101, Oct. 2004.

[13] Y. Yang and T. S. P. Yum, "Maximally flexible assignment of orthogonal variable spreading factor codes for multirate traffic," IEEE Trans. Wireless Commun., vol. 3, pp. 781-792, May 2004.

[14] L. Liu and F. Adachi, "Chip-interleaved multi-rate CDMA with 2dimensional OVSF spreading," in Proc. IEEE VTC 2006-Spring, vol. 4, pp. 1767-1771, May 2006.

[15] K. Hasegawa, R. Shimura, and I. Sasase, "OVSF code allocation and two-stage combining method to reduce intercode interference in OFCDM system," Electron. and Commun. in Japan, Part 1, vol. 90, no. 9, pp. 1623, 2007.

[16] L. Fang and L. B. Milstein, "Successive interference cancellation in multicarrier DS/CDMA," IEEE Trans. Commun., vol. 48, no. 9, pp. 15301540, Sept. 2000.

[17] W. C. Y. Lee, Mobile Communications Engineering: Theory and Applications, 2nd edition. New York: McGraw-Hill, 1997.

[18] Y. Q. Zhou, J. Wang, and M. Sawahashi, "Downlink transmission of broadband OFCDM systems-part I: hybrid detection," IEEE Trans. Commun., vol. 53, pp. 718-729, Apr. 2005.

[19] —, "Downlink transmission of broadband OFCDM systems-part II: effect of Doppler shift," IEEE Trans. Commun., vol. 54, pp. 1097-1108, June 2006.

[20] H. Atarashi and M. Sawahashi, "Investigation of inter-carrier interference due to Doppler spread in OFCDM broadband packet wireless access," IEICE Trans. Commun., special issue on software defined radio technologies and its applications, vol. 86-B, 2002SRP-28, pp. 291-299, Jan. 2003.

[21] R. B. Ertel and J. H. Reed, "Generation of two equal power correlated Rayleigh fading envelopes," IEEE Commun. Lett., vol. 2, pp. 276-278, Oct. 1998.

[22] Z. Xing, "Parallel ensemble Monte Carlo for device simulation," in Proc. Workshop on High Performance Computing Activities in Singapore, National Supercomputing Research Center, Sept. 1995.

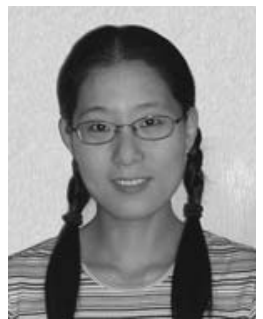

Yiqing Zhou (S'03, M'05) received the B.S. degree in communications and information engineering and the M.S. degree in signal and information processing from the Southeast University, Nanjing, China, in 1997 and 2000, respectively. In February 2004, she received the Ph.D. degree in electrical and electronic engineering from the University of Hong Kong, Hong Kong. Since June 2004, she has been with the Department of Electrical and Electronic Engineering at the University of Hong Kong as a Postdoctoral Fellow. Her research interests include coding theory, spread spectrum, OFDM systems, interference cancellation, hybrid ARQ, MIMO and other transmission techniques for wireless high speed data communications.

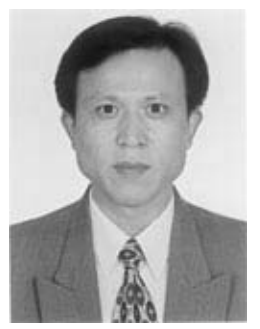

Jiangzhou Wang (M'91, SM'94) received the B.S. and M.S. degrees from Xidian University, Xian, China, in 1983 and 1985, respectively, and the Ph.D. degree (with Greatest Distinction) from the University of Ghent, Belgium, in 1990, all in electrical engineering.

Dr. Wang is currently a Professor and Chair in the Department of Electronics, the University of Kent, England, United Kingdom. From 1995 to 2005, he was with the University of Hong Kong, where he is still serving as an Honorary Professor. From 1992 to 1995, he was a Senior System Engineer at Rockwell International Corporation (now Conexant), California, USA. From 1990 to 1992, he was a Postdoctoral Fellow in the University of California at San Diego, USA. Dr. Wang has held a Visiting Professor position in NTT DoCoMo, Japan. He was a Technical Chairman of IEEE Workshop in 3G Mobile Communications in 12/2000. He has been a technical committee member and session chair of a number of international conferences. Dr. Wang has published over 140 papers, including more than 40 IEEE transactions/journal papers in the areas of wireless mobile and spread spectrum communications. He has written/edited three books, entitled High Speed Wireless Communications (Cambridge University Press, UK, 2/2008), Broadband Wireless Communications (Kluwer, Boston, USA, 6/2001) and Advances in $3 G$ Enhanced Technologies for Wireless Communications (Artech House, Norwood, USA, 2/2002), respectively. The last book has been translated into Chinese.

Dr. Wang is an Editor for IEEE TRANSACTIONS ON COMMUNICATIONS and a Guest Editor of three times for IEEE JOURNAL ON SELECTED AREAS IN COMMUNiCATIONS (Wideband CDMA, 8/2000 and 1/2001, and Advances in Multicarrier CDMA, 6/2006). He holds one US patent in the GSM system. $\mathrm{He}$ is an Evaluation Expert of the European Commission Framework Program 7 (FP7). He is listed in Who's Who in the World.

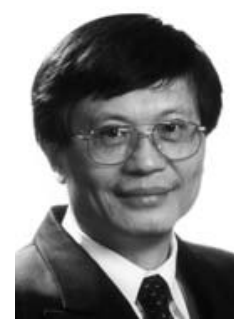

Tung-Sang Ng received the B.Sc.(Eng.) degree from The University of Hong Kong in 1972, the M.Eng.Sc. and Ph.D. degrees from the University of Newcastle, Australia, in 1974 and 1977, respectively, all in electrical engineering.

He worked for BHP Steel International and The University of Wollongong, Australia after graduation for 14 years before returned to The University of Hong Kong in 1991, taking up the position of Professor and Chair of Electronic Engineering. He was Head of Department of Electrical and Electronic Engineering from 2000 to 2003 and Dean of Engineering from 2003 to 2007. His current research interests include wireless communication systems, spread spectrum techniques, CDMA and digital signal processing. He has published over 250 international journal and conference papers.

He was the General Chair of ISCAS'97 and the VP-Region 10 of IEEE CAS Society in 1999 and 2000. He was an Executive Committee Member and a Board Member of the IEE Informatics Divisional Board (1999-2001) and was an ordinary member of IEE Council (1999-2001).

He was awarded the Honorary Doctor of Engineering Degree by the University of Newcastle, Australia in 1997, the Senior Croucher Foundation Fellowship in 1999, the IEEE Third Millenium medal in 2000 and the Outstanding Researcher Award by The University of Hong Kong in 2003. $\mathrm{He}$ is a Fellow of IEEE, IEE and HKIE. 